\title{
Extended cusp-like regions and their dependence on the Polar orbit, seasonal variations, and interplanetary conditions
}

Article

Published Version

Stubbs, T. J., Cargill, P. J., Lockwood, M., Grande, M., Kellett, B. J. and Perry, C. H. (2004) Extended cusp-like regions and their dependence on the Polar orbit, seasonal variations, and interplanetary conditions. Journal of Geophysical Research, 109 (A9). A09210. ISSN 0148-0227 doi:

https://doi.org/10.1029/2003JA010163 Available at https://centaur.reading.ac.uk/38598/

It is advisable to refer to the publisher's version if you intend to cite from the work. See Guidance on citing.

Published version at: http://dx.doi.org/10.1029/2003JA010163

To link to this article DOI: http://dx.doi.org/10.1029/2003JA010163

Publisher: American Geophysical Union

All outputs in CentAUR are protected by Intellectual Property Rights law, including copyright law. Copyright and IPR is retained by the creators or other copyright holders. Terms and conditions for use of this material are defined in the End User Agreement. 


\section{CentAUR}

Central Archive at the University of Reading

Reading's research outputs online 


\title{
Extended cusp-like regions and their dependence on the Polar orbit, seasonal variations, and interplanetary conditions
}

\author{
T. J. Stubbs, ${ }^{1,2}$ P. J. Cargill, ${ }^{1}$ M. Lockwood, ${ }^{3}$ M. Grande, ${ }^{3}$ B. J. Kellett, ${ }^{3}$ and C. H. Perry ${ }^{3}$ \\ Received 24 July 2003; revised 17 May 2004; accepted 24 June 2004; published 25 September 2004.
}

[1] Extended cusp-like regions (ECRs) are surveyed, as observed by the Magnetospheric Ion Composition Sensor (MICS) of the Charge and Mass Magnetospheric Ion Composition Experiment (CAMMICE) instrument aboard Polar between 1996 and 1999. The first of these ECR events was observed on 29 May 1996, an event widely discussed in the literature and initially thought to be caused by tail lobe reconnection due to the coinciding prolonged interval of strong northward IMF. ECRs are characterized here by intense fluxes of magnetosheath-like ions in the energy-per-charge range of $\sim 1$ to $10 \mathrm{keV} \mathrm{e}^{-1}$. We investigate the concurrence of ECRs with intervals of prolonged (lasting longer than 1 and 3 hours) orientations of the IMF vector and high solar wind dynamic pressure $\left(\mathrm{P}_{\mathrm{SW}}\right)$. Also investigated is the opposite concurrence, i.e., of the IMF and high $\mathrm{P}_{\mathrm{SW}}$ with ECRs. (Note that these surveys are asking distinctly different questions.)

The former survey indicates that ECRs have no overall preference for any orientation of the IMF. However, the latter survey reveals that during northward IMF, particularly when accompanied by high $\mathrm{P}_{\mathrm{SW}}$, ECRs are more likely. We also test for orbital and seasonal effects revealing that Polar has to be in a particular region to observe ECRs and that they occur more frequently around late spring. These results indicate that ECRs have three distinct causes and so can relate to extended intervals in (1) the cusp on open field lines, (2) the magnetosheath, and (3) the magnetopause indentation at the cusp, with the latter allowing magnetosheath plasma to approach close to the Earth without entering the magnetosphere. INDEX TERMS: 2724 Magnetospheric Physics: Magnetopause, cusp, and boundary layers; 2784 Magnetospheric Physics: Solar wind/magnetosphere interactions; 2728 Magnetospheric Physics: Magnetosheath; 7835 Space Plasma Physics: Magnetic reconnection; 2164 Interplanetary Physics: Solar wind plasma; KEYWORDS: solar wind plasma, interplanetary magnetic fields, magnetic reconnection, solar wind/magnetosphere interaction, magnetosheath, magnetopause

Citation: Stubbs, T. J., P. J. Cargill, M. Lockwood, M. Grande, B. J. Kellett, and C. H. Perry (2004), Extended cusp-like regions and their dependence on the Polar orbit, seasonal variations, and interplanetary conditions, J. Geophys. Res., 109, A09210, doi:10.1029/2003JA010163.

\section{Introduction}

[2] The cusp regions are known to be important in understanding the transfer of mass, energy, and momentum from the solar wind to the magnetosphere. As a consequence, we also need to understand the dynamics and structure of the cusp and its dependence on internal and external influences. The low-altitude cusp has been studied extensively [e.g., Newell and Meng, 1988] and statistical surveys show it to be located around local noon and to vary in latitude depending on the time of year and the orientation of the interplanetary magnetic field (IMF).

\footnotetext{
${ }^{1}$ Space and Atmospheric Physics, Blackett Laboratory, Imperial College, London, UK

${ }^{2}$ Now at Laboratory for Extraterrestrial Physics, NASA Goddard Space Flight Center, Greenbelt, Maryland, USA.

${ }^{3}$ Space Science Department, Rutherford Appleton Laboratory, Oxfordshire, UK.

Copyright 2004 by the American Geophysical Union. 0148-0227/04/2003JA010163\$09.00
}

For southward IMF the occurrence and structure of the cusp is well explained by magnetopause reconnection between the magnetosheath field and closed geomagnetic field lines and its variations in space and time [Lockwood, 1995a, 1995b]. The Polar spacecraft explores the more dynamic midaltitude cusp region at radial distances of between 6 and $9 \mathrm{R}_{\mathrm{E}}$. It is here that extended cusp-like regions are observed with the Magnetospheric Ion Composition Sensor (MICS) of the Charge and Mass Magnetospheric Ion Composition Experiment (CAMMICE) instrument, as first reported by Grande et al. [1997] and Chen et al. [1997]. Cusp ions have energies below $\sim 10$ $\mathrm{keV}$, and extended cusp-like regions, hereafter referred to as ECRs, are characterized in the present study by intense fluxes of solar wind/magnetosheath-like ions in the energyper-charge range of around 1 to $10 \mathrm{keV} \mathrm{e}^{-1}$ which are observed for periods longer than $30 \mathrm{~min}$. Grande et al. [1997] discussed an ECR observed on 29 May 1996 by Polar and other spacecraft. This particular ECR coincided with a prolonged interval of strong northward IMF and had angular distributions in the 1 to $\sim 30 \mathrm{keV}$ protons 
that showed evidence of flows and trapping. Therefore it was suggested that reconnection had occurred in the tail lobe, resulting in an extended region of magnetosheath ion fluxes at high invariant latitudes. From previous observations and models of the magnetospheric magnetic field, such significant quantities of plasma of solar wind origin were not expected to be able to gain access to the midaltitude dayside magnetosphere [Russell et al., 1998].

[3] More recently, work by Onsager et al. [2001], using electron and proton distributions from Hydra, has identified regions within the 29 May 1996 event associated with high-latitude lobe reconnection. They argued that for most of the event, Polar was predominantly in the magnetosheath, as evidenced by relatively low electron fluxes at energies above $\sim 200 \mathrm{eV}$. One of the most consistent indicators of the magnetopause location during low magnetic shear is an anisotropy in electron temperature [Paschmann et al., 1993]. Onsager et al. used this indicator to differentiate between electron heating parallel and antiparallel to the magnetic field, thus allowing them to ascertain whether lobe reconnection had occurred in either the northern or southern hemisphere or both. Unlike the previous studies discussed, these arguments were based strongly on the electron distributions. This result was reconciled with previous observations and interpretation [e.g., Chandler et al., 1999] by suggesting that some preprocessing mechanisms act on the magnetosheath in the region of the high-latitude cusp, thus accounting for the slow bulk speed and the structure of the ion distributions.

[4] Stubbs et al. [2000] studied an ECR event on 14 August 1996 that coincided with an interval of weak southward IMF, thus showing that ECRs did not occur only during strongly northward IMF conditions. They also compared MFE magnetic field data with the Tsyganenko [1989] magnetic field (T89) model and showed that the observed magnetic field was much weaker than predicted during ECR events. A similar discrepancy using the Tsyganenko [1996] (T96) model was discussed by Tsyganenko and Russell [1999], who surveyed the magnetic cusp signatures observed by Polar between 1996 and 1998 .

[5] Understanding the behavior of ECRs and their dependency on external conditions is key to understanding the underlying physics of how these regions are formed. Here we present studies of (1) how ECRs are distributed in time and space throughout the period from March 1996 to June 1999; (2) the coincidence of ECR events with prolonged periods of the IMF orientation along each axial direction of the GSM coordinate system, as well as with prolonged intervals of high solar wind dynamic pressure; and (3) the coincidence of intervals in the solar wind with ECRs, a quite different question. We begin with a description in section 2 of the spacecraft and data sets used in this work. In section 3 we define an ECR and describe our selection criteria. This is followed in section 4 by the results of our statistical studies. Section 5 is a discussion of the results and how they relate to previous studies (in particular that by Chen et al. [1998]). Section 6 is our summary and conclusions. To study the ECR dependencies on interplanetary conditions, we needed to develop a code to calculate the propagation time of the solar wind plasma observed by the
Wind and ACE spacecraft in interplanetary space to Polar in the cusp. The assumptions and methods used in this code are described in Appendix A.

\section{Spacecraft and Instrumentation}

[6] The Polar spacecraft is in a $1.8 \times 9 \mathrm{R}_{\mathrm{E}}$ polar orbit with an inclination of $\sim 86^{\circ}$ to the equator, as shown in Figure 2, and a period of $\sim 17.5$ hours [Acuña et al., 1995]. Its apogee is at high northern latitudes and it is spinstabilized at $10 \mathrm{rpm}$ with its spin axis approximately perpendicular to the orbital plane. In this study we use data from the CAMMICS MICS instrument. The Electrostatic Analyzer (ESA) on MICS sorts by energy-per-charge (E/q) and has 25 operational channels with midpoints ranging from 1.0 to $193.4 \mathrm{keV} \mathrm{e}^{-1}$. Sorting by the ESA, combined with time-of-flight measurements and determination of the energy deposited in the solid state detector, allows MICS to determine the flux, mass, and charge states of the incident ions [Wilken et al., 1992; Fritz et al., 1997]. MICS has a temporal resolution of $197.5 \mathrm{~s}$. We also used data from the Magnetic Field Experiment (MFE) aboard Polar, which has been averaged over $55.2 \mathrm{~s}$ [Russell et al., 1995].

[7] For the period from 18 March 1996 until 28 February 1998, we used data from the Wind spacecraft to monitor interplanetary conditions [Acuña et al., 1995]. After these times we switched to using data from the ACE spacecraft [Stone et al., 1998], as the Wind orbit began to frequently enter the magnetosphere and reach distances far from the Sun-Earth line, whereas ACE remained around the L1 point upstream of the Earth. From Wind, magnetic field data was taken from MFI (Magnetic Fields Investigation) at 46-s resolution [Lepping et al., 1995], and the solar wind plasma moments were generated from SWE (Solar Wind Experiment) at 92-s resolution [Ogilvie et al., 1995]. From ACE, magnetic field data was taken from MAG (Magnetic Fields Experiment) at 16-s resolution [Smith et al., 1998], and the plasma moments were taken from SWEPAM (Solar Wind Electron Proton Alpha Monitor) at 64-s resolution [McComas et al., 1998]. Our processing of Wind and ACE data is discussed in Appendix A.

\section{Definition of an Extended Cusp-Like Region}

[8] The ECRs are identified using data from both the CAMMICE MICS and MFE instruments. As stated earlier, we define ECRs as being primarily characterized by intense fluxes of solar wind/magnetosheath-like ions in the $\mathrm{E} / \mathrm{q}$ range from around 1 to $10 \mathrm{keV} \mathrm{e}^{-1}$ observed for periods longer than 30 min by MICS. (If there is a gap of less than 15 min between ECRs, then they are merged and counted as one ECR event. This crude combination is necessary as it can be difficult to be consistent in distinguishing individual events.) Magnetosheath plasma has lower energies than magnetospheric plasma and a significant population of high charge state species such as $\mathrm{He}^{2+}$ and $\mathrm{O}>{ }^{2+}$, whereas magnetospheric plasma tends to contain low charge state ion species such as $\mathrm{He}^{+}$and $\mathrm{O}^{+}$, indicative of their terrestrial origin. This charge state transition can be seen in Figures 3a to 3d of Grande et al. [1997] at around 0300 UT. We also attempt to distinguish between the low-latitude boundary layer (LLBL) and cusp, but it is not always clear where the 
LLBL ends and the cusp begins in MICS data. The LLBL is a mixture of high-energy magnetospheric plasma and the high-energy component of lower-energy magnetosheath plasma that has crossed the magnetopause [Lockwood and Smith, 1992]. For an "open LLBL" (also called the reconnection layer), the magnetospheric ions are at an energy below the time-of-flight cut-off energy, $\mathrm{E}_{\mathrm{ic}}$, for a given position and pitch angle, whereas the magnetosheath ions are at energies above $\mathrm{E}_{\text {ic }}$ [Lockwood, 1997]. Hence energy ranges which span $E_{\text {ic }}$ and/or which average over a wide range of pitch angles will contain a mixture of these two populations. The cusp is assumed to constitute magnetospheric field lines where plasma of magnetosheath origin dominates. The magnetic field data can be used to aid the identification of the boundary between the magnetosphere and the ECR. In the magnetic field data this is seen as a transition from an ordered, steady closed geomagnetic field to disturbed field lines connected to the magnetosheath and populated with shocked magnetosheath plasma [e.g., see Russell et al., 1998]. The difference between the observed magnetic field and the predicted T89 field, $\Delta \mathrm{B}$, is also useful as it is typically large and negative in the cusp region [Farrell and Van Allen, 1990].

[9] At energies-per-charge between 1 and $10 \mathrm{keV} \mathrm{e}^{-1}$, we are in fact observing the high-energy tail of the cusp ion distribution. Hydra plasma observations at thermal energiesper-charge between $\sim 2 \mathrm{eV}$ and $35 \mathrm{keV} \mathrm{e}^{-1}$, which measures most of the cusp phase space density, have shown that Polar can be in the cusp region without MICS observing any magnetosheath particles [Lockwood et al., 2001]. As discussed later, the MICS definition of cusp particles often appear to correspond to the part of the cusp on more recently reconnected field lines, closer to the reconnection site. Therefore the observational criteria for an ECR are not the same as those for the cusp. The advantage of using MICS for such studies over Hydra is the ability to distinguish charge state, thus allowing us to determine the source of the plasma.

[10] This definition of an ECR concentrates on the source of the plasma in the dayside magnetosphere. The analysis by Onsager et al. [2001] suggests that even some magnetically well-ordered ECR events we have identified with MICS may well include traversals into the magnetosheath. However, this does not affect the question of how magnetosheath plasma was observed in such significant quantities in the region currently regarded as the midaltitude cusp.

[11] The ECR events described here are similar to the cusp energetic particle (CEP) events and cusp diamagnetic cavities (CDCs) previously studied by Chen et al. [1998] and Fritz et al. [2003]. In section 5 we describe these events and how they relate to our work here.

\section{Method and Results}

[12] CAMMICE MICS observed 191 ECRs from 18 March 1996 until 30 June 1999; during this period MICS experienced very few data gaps, therefore we can be reasonably sure that few, if any, of the ECRs traversed by Polar were missed by this survey. One hundred eighty-four out of the 191 ECRs observed by MICS are used in the concurrence surveys (over 96\%). Seven ECRs were not used, as they typically had less than $20 \%$ solar wind data coverage, while the remaining events had at least $90 \%$ solar wind data coverage. The 184 ECRs used in the concurrence studies total almost 443 hours of observation time with an average duration of $\sim 2.4$ hours.

[13] We begin by investigating how the ECRs are distributed in space $(\Lambda$, MLT, and R) and use this to define the region where ECRs are observed, which is later used in the coincidence studies. We then look at how ECR occurrences are distributed throughout the 3.25 years of observations. This is followed by analysis of the coincidence of ECRs with both prolonged orientations of the IMF vector in GSM coordinates and intervals of prolonged high solar wind dynamic pressure $\left(\mathrm{P}_{\mathrm{SW}}\right)$. We also examine the opposite coincidence, namely of prolonged orientations of the IMF vector and high $\mathrm{P}_{\mathrm{SW}}$ with ECRs. It must be appreciated that these surveys are asking distinctly different questions.

\subsection{Distribution of Extended Cusp-Like Regions}

[14] As we would expect, the ECRs are distributed around noon in magnetic local time (MLT) and at invariant latitudes $(\Lambda)$ around $80^{\circ}$, as shown by Figure 1a, and centered around geocentric distances $(R)$ of $8 R_{E}$ (Figure $1 b$ ). The gray lines are the 184 ECR trajectories used in the concurrence studies, and the black lines are the seven not used due to a lack of accompanying solar wind data. These figures clearly show a preference in the occurrence of ECRs to certain ranges of invariant latitude, magnetic local time, and geocentric distance.

[15] In the concurrence studies, we will only consider prolonged intervals of IMF orientation or $\mathrm{P}_{\mathrm{SW}}$ that overlap with times when Polar is in the region where ECRs are expected to be observed. This "ECR observation region" is defined in these studies as lying within the dark gray dashed ellipse in Figure 1a and above the dark gray dashed curve in Figure 1b. The ellipse in Figure 1a is centered at $\Lambda=81.8^{\circ}$ and 1103 MLT, and the curve in Figure $1 \mathrm{~b}$ is at $\mathrm{R}=6.25 \mathrm{R}_{\mathrm{E}}$. The ECR observation region fully encompasses 172 out of 191 ECR trajectories ( 90\%). Of the remaining 19 ECRs, six fall entirely outside of the ECR observation region $(\sim 3 \%)$. By doing this, we have managed to discard the vast majority of the Polar orbit that is unsuitable for ECR observations. We set the condition that Polar has to be within the ECR observation region for at least $30 \mathrm{~min}$ (i.e., the minimum duration of an ECR) for an interval to be included in the concurrence studies.

[16] We also set the criteria that an interval of solar wind data (time lagged to the Earth using the method described in Appendix A) coinciding with a time when Polar is entering the ECR observation region must overlap by at least $30 \mathrm{~min}$. Similarly, an interval of solar wind data must overlap by at least 45 min when Polar is leaving the ECR observation region. This is to ensure that the selected intervals in the solar wind actually affect the likelihood of observing an ECR. The overlap for intervals in the solar wind data coinciding with Polar leaving the ECR observation region are required to be longer because of the finite time taken for the magnetosphere to respond to changes in the solar wind.

[17] The orbit of Polar is approximately fixed in inertial space; therefore relative to the Sun it performs a complete rotation about an axis normal to the ecliptic plane every year, so there will be a preferred time of the year for sampling the ECR observation region. As the orbital plane 

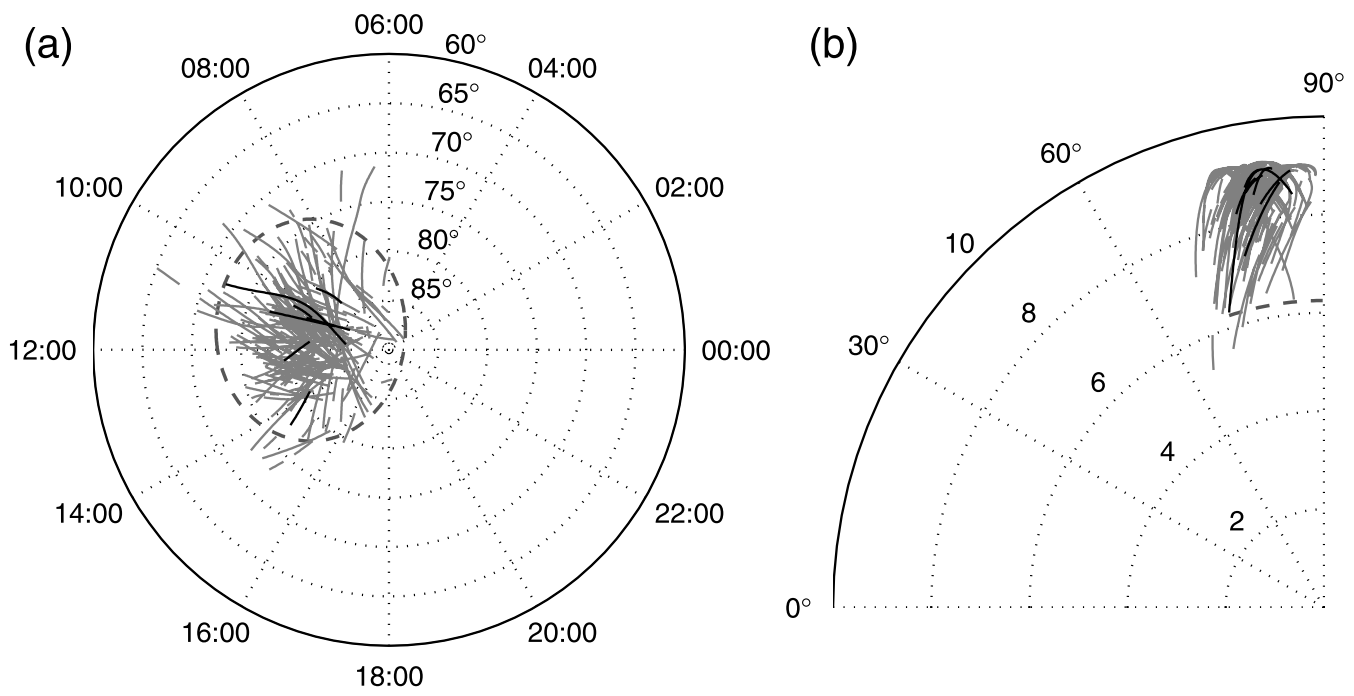

Figure 1. Polar trajectories during the 184 ECRs used in the concurrence studies (gray solid lines) and the seven ECRs not used due to lack of accompanying solar wind data (black solid lines). (a) Trajectories plotted as invariant latitude $(\Lambda)$ versus magnetic local time (MLT). The dark gray elliptical dashed line centered at $\Lambda=81.8^{\circ}$ and 1103 MLT encompasses the majority of ECR trajectories (172 out of 191 or $\sim 90 \%$ ) and effectively defines where we expect to observe ECRs. (b) Trajectories plotted as $\Lambda$ versus geocentric radius $(\mathrm{R})$. The dark gray dashed line at $\mathrm{R}=6.25 \mathrm{R}_{\mathrm{E}}$ is the minimum radius at which we typically expect to observe ECRs.

is inclined to the equator, it tilts toward dusk in spring and toward dawn during the fall (northern hemisphere seasons), as shown in Figure $2 \mathrm{~b}$. The apogee of the orbit is also precessing in the manner shown in Figure $2 \mathrm{a}$, such that it usually faces toward the Sun in the spring and away from the Sun in the fall.

[18] The precession of the orbit apogee is also evident in Figure 3, where we have plotted the Polar trajectories during ECR events as a function of $\mathrm{X}$ and $\left(\mathrm{Y}^{2}+\mathrm{Z}^{2}\right)^{1 / 2}$ in GSM coordinates. (Note that both $\mathrm{X}$ and $\left(\mathrm{Y}^{2}+\mathrm{Z}^{2}\right)^{1 / 2}$ will have the same value if plotted using GSE coordinates.) Again, the gray lines represent the 184 ECRs used in the concurrence studies and the black lines represent the remaining seven events. Predictions from the empirical magnetopause model of Sibeck et al. [1991] are shown by the dark gray lines for a solar wind dynamic pressure $\mathrm{P}_{\mathrm{SW}}$ of $1.0 \mathrm{nPa}$ (dashed), $3.0 \mathrm{nPa}$ (solid), $7.0 \mathrm{nPa}$ (dotted), and $12.0 \mathrm{nPa}$ (dash-dot). Note that the mode value of the distribution of $\mathrm{P}_{\mathrm{SW}}$ values is $3 \mathrm{nPa}$ [Hapgood et al., 1991], and thus the last two curves are for above average pressure and the last is for decidedly rare conditions. The Sibeck et al. [1991] model in this case is self-similar, as it does not change shape with changing $\mathrm{P}_{\mathrm{SW}}$ and is symmetric about the GSM X-axis. We can see that events in 1996 and 1997 at low values of X are well within the predicted magnetopause boundaries. As the orbit apogee precessed toward the equator, in the spring some ECR events began to approach the predicted magnetopause boundary for $\mathrm{P}_{\mathrm{SW}}=7.0 \mathrm{nPa}$. It is possible that ECR events associated with higher $\mathrm{P}_{\mathrm{SW}}$ crossed the magnetopause, particularly during 1999. The Sibeck et al. [1991] model used here does not include the effects of flux erosion from the subsolar and tail lobe magnetopause for southward and northward IMF, respectively. The IMF can alter the location of the magnetopause boundary, possibly resulting in Polar entering the magnetosheath. By using this model, we have concentrated on the effects of $\mathrm{P}_{\mathrm{SW}}$ on the location of the magnetopause.

[19] Figure 4 shows the monthly statistics for ECR occurrences and Polar sampling, where the dark gray bars and lines represent all times when MICS data was available, while the light gray bars and lines are for times with accompanying solar wind data. When commenting on features and trends in the data, we shall refer to the data for all times when MICS data was available. We note that the ECRs used in the concurrence studies (light gray bars and lines) are representative of all MICS data. The most apparent difference is the peak occurrence of 1998 happening in April instead of May.

[20] Figures $4 \mathrm{a}$ and $4 \mathrm{~b}$ show the number of ECRs observed per month and the number of hours of ECR observations per month, respectively. Both figures show ECR occurrences grouping around particular months of the year. In Figure 4a we can see that in 1996 the ECR occurrences peak in September and in 1997 they peak in April with a small recurrence in September and October. In 1998 and 1999 the occurrences peak in May.

[21] Figures 4c, 4d, and 4e show the number of traversals of the ECR observation region (as defined by the dark gray dashed lines in Figure 1), the number of hours spent in this region, and the total number of hours of sampling over the entire magnetosphere, respectively. As expected, the sampling of the ECR observation region, shown in Figure 4d, roughly follows the same trend as the ECRs in Figures $4 \mathrm{a}$ and $4 \mathrm{~b}$. This trend is even more apparent in the number of hours in the ECR observation region (Figure 4d). From Figure $4 \mathrm{e}$, we see that the data coverage was typically very good, rarely falling below 625 hours per month. The horizontal black dotted line indicates the 30-day mark, which is around the value we would expect the data to have for full coverage. 

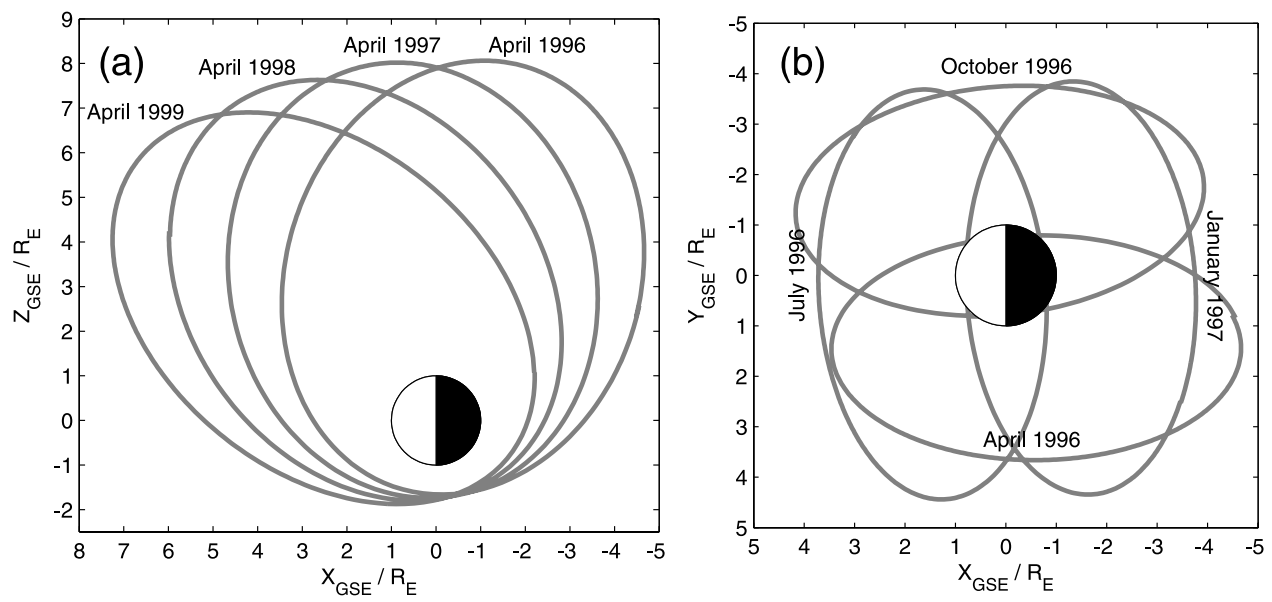

Figure 2. (a) The precession of the orbit toward the Sun in spring, shown here for 15 April from 1996 to 1999. (b) Tilt of the orbital plane at different times of the year, shown here for 15 April 1996, 15 July 1996, 15 October 1996, and 15 January 1997.

[22] Finally, we try to normalize the ECR occurrences by calculating the occurrence frequencies. Figure $4 \mathrm{f}$ shows the ratio of the number of ECR observations to the number of ECR observation region traversals, while Figure $4 \mathrm{~g}$ shows the ratio of the number of hours of ECR observation to the time spent in the ECR observation region. From Figure 4f, we can see that occurrence frequencies peak during September 1996, April 1997, April 1998, and May 1999 with the overall peak in April 1998. The black line in Figure 4f represents the ECR occurrence per orbit, i.e., the occurrence frequency for all available MICS data corrected for multiple ECRs per orbit (so the maximum possible occurrence frequency is $100 \%$ ). As expected, it is occasionally lower than those represented by the dark and light gray bars, but overall it is comparable with the other data. Occurrence frequencies shown in Figure $4 \mathrm{~g}$ are broadly consistent with those shown in Figure 4f, with peaks around August 1996, May 1997, May 1998, and April 1999, and the overall peak in May 1998. The occurrence frequencies during September and October 1997 are small, suggesting a genuinely low occurrence.

[23] From Figure $4 \mathrm{f}$ we can see that ECR occurrence rates are $25 \%, 35 \%, 68 \%$, and $43 \%$ for September 1996, April 1997, April 1998, and May 1999, respectively. Therefore it appears that under suitable conditions, such as those prevalent around April 1998, Polar is able to observe ECRs on the majority of orbits. (From the data in Figure $4 \mathrm{~g}$ it can be shown that the occurrences in April and May of 1998 and 1999 are almost 3 standard deviations above the mean, thus indicating that they are extremely statistically significant.) Also, from a comparison of the time of year of the peak ECR occurrence rates, we notice that they are generally higher when observed in the spring than in the fall, as shown by comparing observations in the spring of 1997 to 1999 with the fall of 1996 and 1997. This could possibly be due to seasonal variation in the cusp and ECR location, as well as orbital sampling.

\subsection{Coincidence of ECRs With Interplanetary Conditions}

[24] In this section we discuss the coincidence of ECRs with both prolonged orientations of the IMF vector in
GSM coordinates and intervals of prolonged high solar wind dynamic pressure. Figure 5 shows the results for intervals of various interplanetary conditions lasting longer than 1 hour (Figures 5a and 5b) and 3 hours (Figures 5c and $5 \mathrm{~d}$ ). Sunward and antisunward correspond to components of the IMF vector parallel and antiparallel to the GSM $\mathrm{X}$-axis, respectively. Likewise, eastward and westward similarly correspond to the GSM Y-axis, and northward and southward correspond to the GSM Z-axis. The Parker

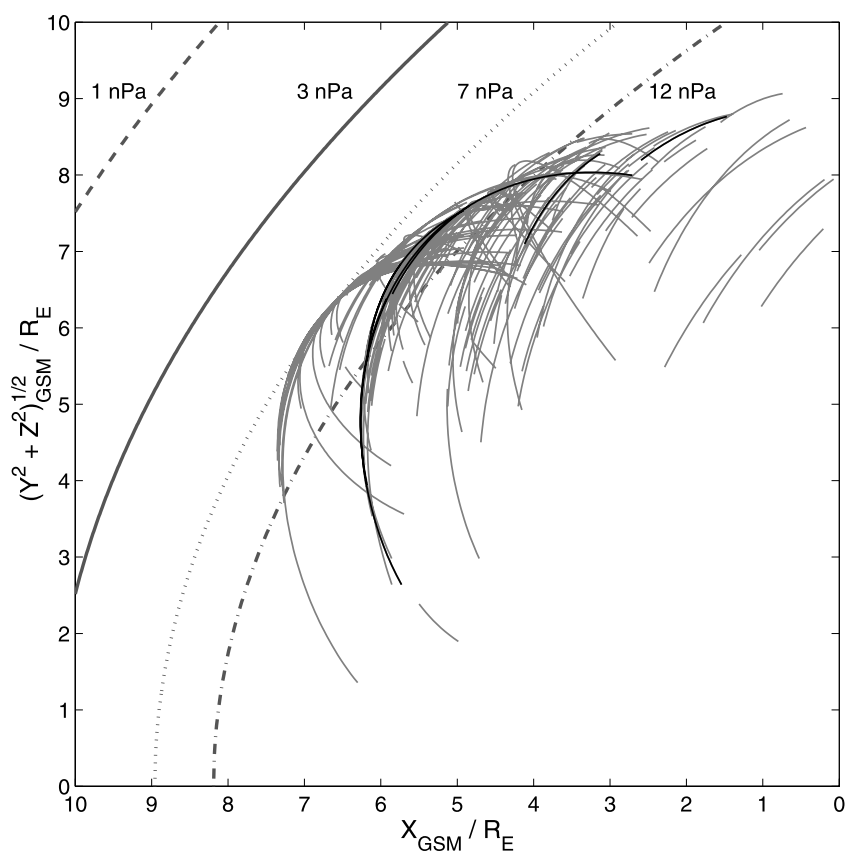

Figure 3. Polar trajectories of the 184 ECRs used in concurrence studies (gray solid lines) and the seven not used (black lines), plotted as $\mathrm{X}$ versus $\left(\mathrm{Y}^{2}+\mathrm{Z}^{2}\right)^{1 / 2}$ in GSM coordinates in units of $\mathrm{R}_{\mathrm{E}}$. These have been plotted with the Sibeck et al. [1991] $\mathrm{P}_{\mathrm{Sw}}$-dependent magnetopause model, where boundaries are represented by the dark gray lines for $1.0 \mathrm{nPa}$ (dashed), $3.0 \mathrm{nPa}$ (solid), $7.0 \mathrm{nPa}$ (dotted), and $12.0 \mathrm{nPa}$ (dash-dot). 
(a)

(b)

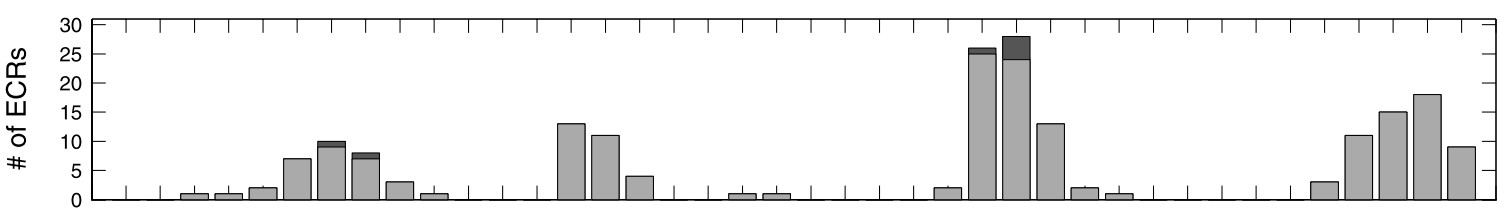

(c)

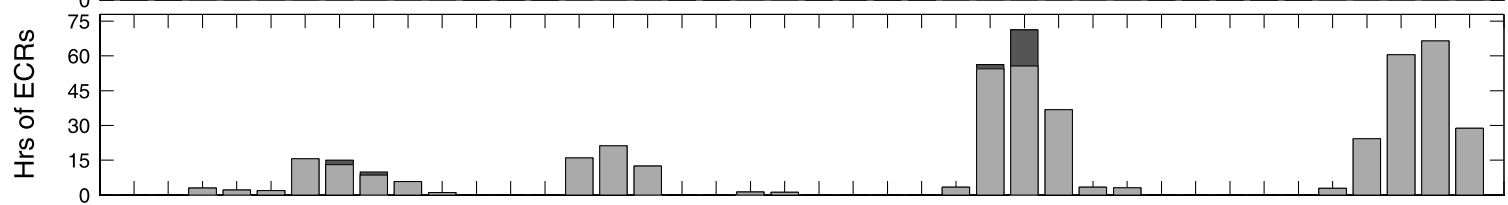

(d)
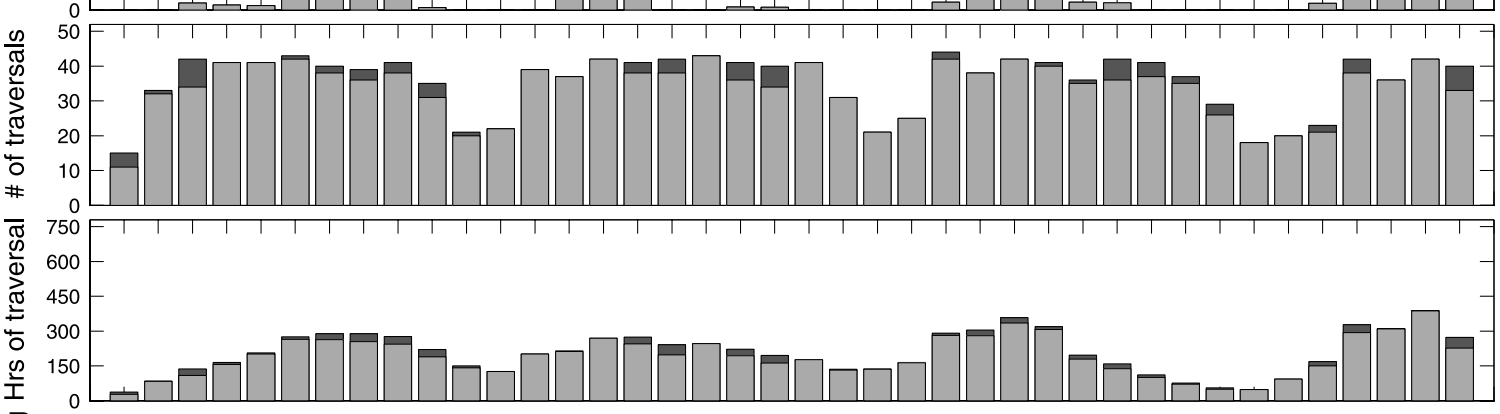

(e)

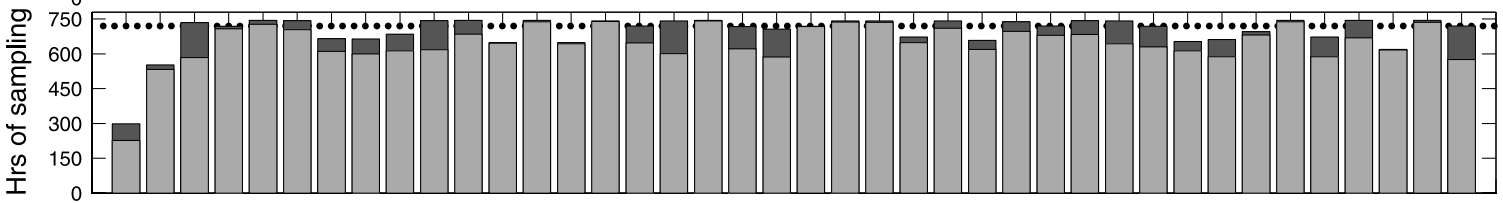

(f)

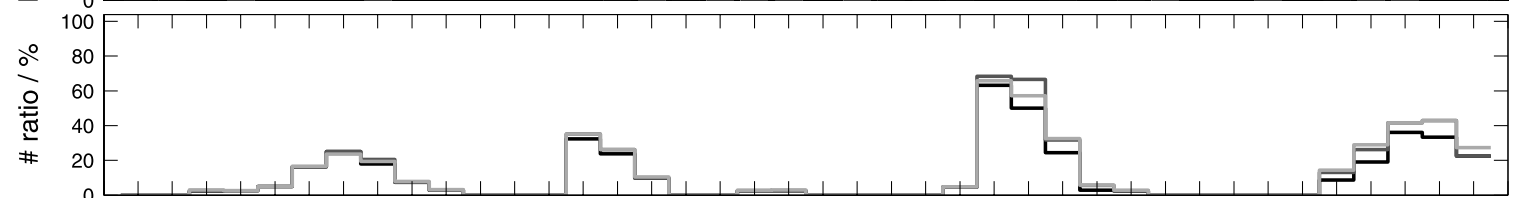

(g)

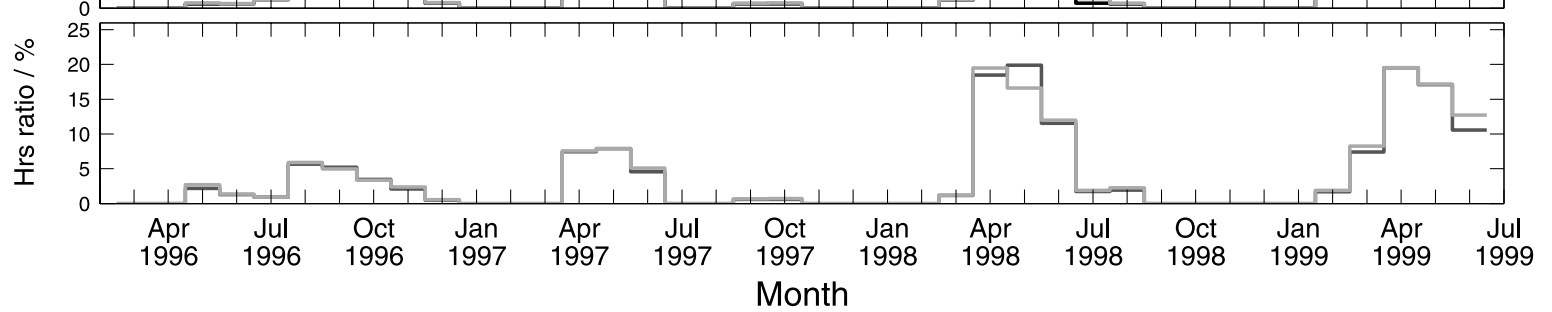

Figure 4. Monthly statistics for ECR occurrences and Polar sampling. The dark gray bars and lines are for all times when MICS data was available; the light gray bars and lines are for times with accompanying solar wind data as well. (a) The number of ECRs observed per month. (b) Number of hours of ECR observation per month. (c) Number of traversals of the ECR observation region, as defined by the dark gray dashed lines in Figure 1. (d) Number of hours spent traversing the ECR observation region. (e) Total hours of CAMMICE MICS sampling along Polar's entire orbit per month, where the horizontal black dotted line marks 30 days (indicates the quality of the overall sampling each month). (f) The ratio of the number of ECRs observed to the number of ECR observation region traversals, where the black line is the occurrence per orbit for all times (i.e., the dark gray line corrected for multiple ECRs during one orbit). (g) The ratio of the number of hours spent observing ECRs to the number spent traversing the ECR observation region.

spiral and ortho-Parker spiral IMF configurations correspond to $\mathrm{B}_{\mathrm{X}} / \mathrm{B}_{\mathrm{Y}}<0$ and $\mathrm{B}_{\mathrm{X}} / \mathrm{B}_{\mathrm{Y}}>0$, respectively. $\mathrm{P}_{\mathrm{SW}}$ is binned for intervals of prolonged magnitude less than $3 \mathrm{nPa}$ and intervals with prolonged magnitude continuously greater than $3 \mathrm{nPa}, 5 \mathrm{nPa}$, and $7 \mathrm{nPa}$. The bins for $\mathrm{P}_{\mathrm{SW}}>5 \mathrm{nPa}$ and $\mathrm{P}_{\mathrm{SW}}>7 \mathrm{nPa}$ are consequently subsets of $\mathrm{P}_{\mathrm{SW}}>3 \mathrm{nPa}$, while $\mathrm{P}_{\mathrm{SW}}>7 \mathrm{nPa}$ is also a subset of $\mathrm{P}_{\mathrm{SW}}>5 \mathrm{nPa}$. An interval in the solar wind is only considered here if it overlaps by at least 5 min with a time when Polar enters an ECR and by at least 15 min with a time when it is leaving an ECR. Again, this is to ensure that the two events are related; this is less likely when Polar is leaving an ECR. As it is possible for a component of the IMF to change from one sense to another during an ECR, the total number of ECRs associated with a particular component of the IMF can exceed $100 \%$ (e.g., the combined coincidence of northward and southward IMF $\approx 107 \%$, as shown in Figure 5 b). From the 29 May 1996 event we would expect the occurrence of 

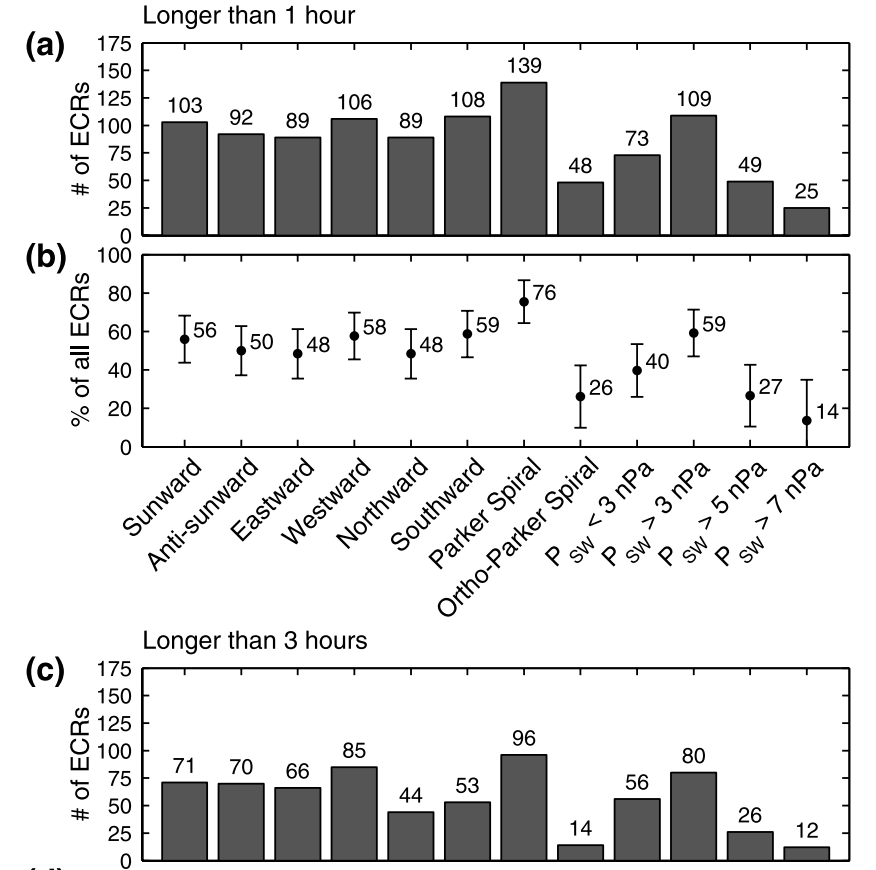

(d)

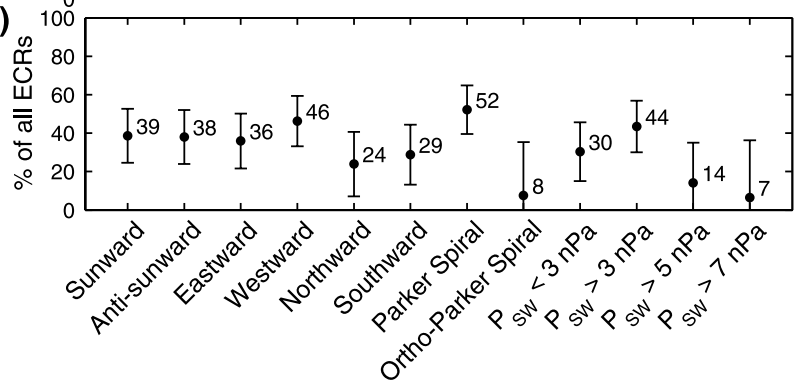

Figure 5. The coincidence of the 184 ECR events with orientations of the IMF vector and intervals of sustained $\mathrm{P}_{\mathrm{SW}}$ lasting longer than 1 hour, as shown in Figures $5 \mathrm{a}$ and $5 \mathrm{~b}$, and lasting longer than 3 hours, as shown in Figures $5 \mathrm{c}$ and 5 d. Figures $5 \mathrm{a}$ and $5 \mathrm{c}$ show the number of ECRs associated with a particular interplanetary condition. Figures $5 \mathrm{~b}$ and $5 \mathrm{~d}$ show the percentage of all ECRs that this represents. Uncertainties are estimated using Poisson counting statistics.

ECRs to favor prolonged periods of northward IMF and high $\mathrm{P}_{\mathrm{SW}}$ above $5 \mathrm{nPa}$.

[25] The error bars in the following figures (e.g., Figures $5 \mathrm{~b}$ and $5 \mathrm{~d}$ ) are calculated using Poisson statistics, i.e., the uncertainty is $\mathrm{N}^{-1 / 2}$, where $\mathrm{N}$ is the number of observations. Therefore the total uncertainty is given to be $\left(1 / \mathrm{N}_{\mathrm{ECRi}}+1 / \Sigma \mathrm{N}_{\mathrm{ECRi}}\right)^{1 / 2}$, where $\mathrm{N}_{\mathrm{ECRi}}$ is the number of ECRs associated with a particular interplanetary condition and $\Sigma \mathrm{N}_{\mathrm{ECRi}}$ is the total number of ECRs observed. On occasion we shall also refer to the number of standard deviations $(\sigma)$ that an occurrence is from the mean occurrence of the distribution, in order to highlight its statistical significance.

[26] Figure 5b shows that all ECRs are associated with at least one interval of prolonged IMF orientation lasting longer than 1 hour. This is not surprising as components of the IMF are often observed to maintain the same orientation on time scales of around an hour. We can also see that ECRs are associated with an approximately even distribution of IMF orientations about each GSM axis to within the given uncertainties $(\sim \pm 15 \%)$. This result is similar to that of Hapgood et al. [1991], whose statistical study of hour-averaged solar wind data over two solar cycles showed that, on average, there is no preferred direction in these components of the IMF. The results in Figure 5 are what one would expect if ECRs occurred randomly relative to interplanetary conditions, as they mirror the occurrence distributions seen in the solar wind. From Figure 5 we also note that negative values of $B_{X} / B_{Y}$, indicating a Parker spiral IMF, are preferred during ECRs by 76 to $26 \%$. This, again, is expected from the study by Hapgood et al. [1991], where IMF $\mathrm{B}_{\mathrm{X}} / \mathrm{B}_{\mathrm{Y}}<0$ was shown to be about three times more likely than IMF $\mathrm{B}_{\mathrm{X}} / \mathrm{B}_{\mathrm{Y}}>0$. ECRs are associated more with intervals of high $\left(\mathrm{P}_{\mathrm{SW}}>3 \mathrm{nPa}\right)$ rather than low $\left(\mathrm{P}_{\mathrm{SW}}<3 \mathrm{nPa}\right)$ solar wind dynamic pressure lasting for an hour or more. The value of $3 \mathrm{nPa}$ was chosen, as it is the mode value for $\mathrm{P}_{\mathrm{SW}}$ [Hapgood et al., 1991]. However, there appears to be no preference shown for intervals of prolonged $\mathrm{P}_{\mathrm{SW}}>5 \mathrm{nPa}$.

[27] From a comparison of Figures 5a and 5c, we notice that ECRs associated with intervals lasting longer than 3 hours typically account for over half of the intervals lasting longer than an hour. Therefore it is not unusual for
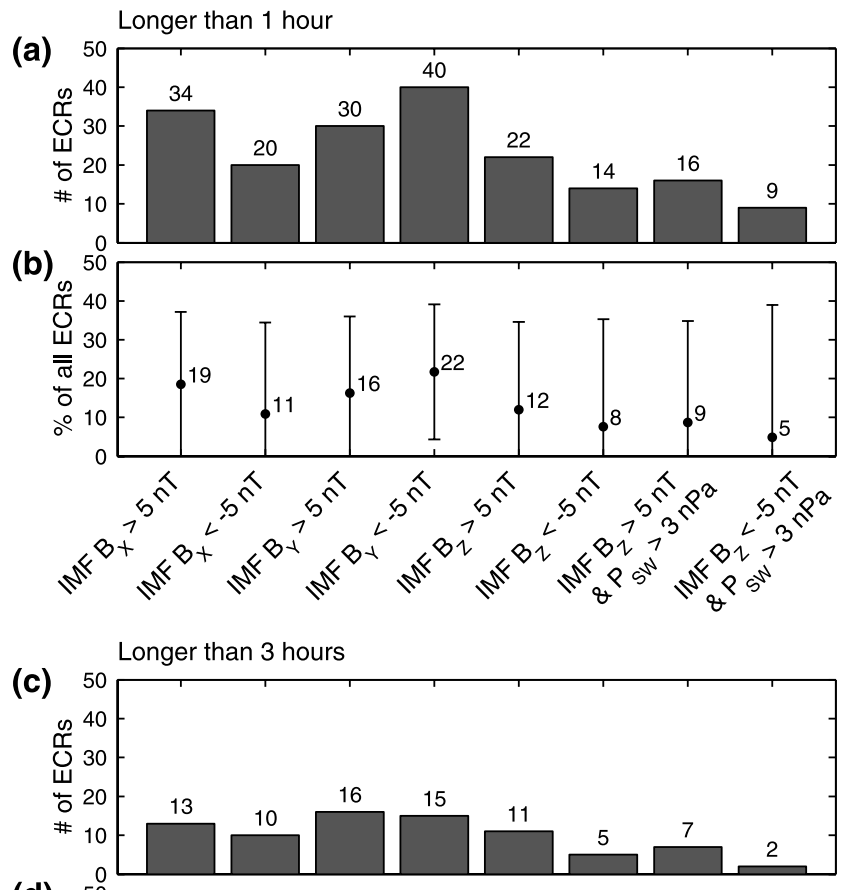

(d)

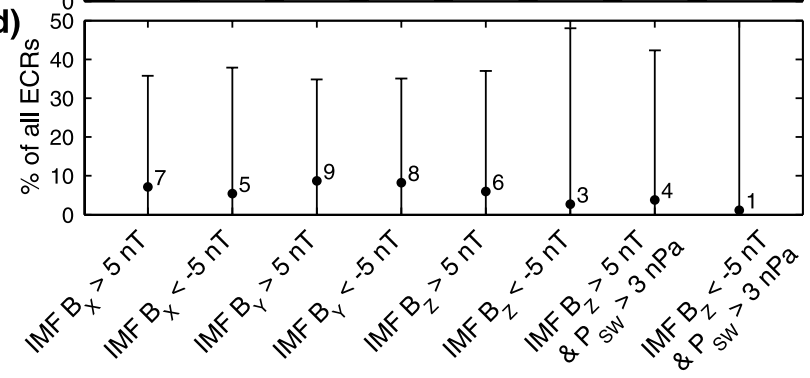

Figure 6. Same format as Figure 5; however, in this case the magnitude of the IMF component must remain above $5 \mathrm{nT}$. Also shown are the coincidences for prolonged intervals of strong magnetic magnitude $(>|5| \mathrm{nT})$ in the IMF $\mathrm{B}_{\mathrm{Z}}$-component coinciding with prolonged high $\mathrm{P}_{\mathrm{SW}}(>3 \mathrm{nPa})$. 
ECRs to coincide with long duration intervals ( $>3$ hours) in the solar wind.

[28] Figure 6 shows the results of a comparison between prolonged intervals of various components of the IMF with a magnitude greater than $5 \mathrm{nT}$. As we can see, the uncertainties are much greater here and no clear preference is shown for any of these conditions, although ECRs do coincide with intervals of IMF $\mathrm{B}_{\mathrm{Y}}<-5 \mathrm{nT}$ and IMF $\mathrm{B}_{\mathrm{X}}>5 \mathrm{nT}$, which last longer than an hour, slightly more often than the other parameters shown. These results certainly do not appear to dominate those shown in Figure 5, i.e., they are a small part of the total sample. We can also see that the intervals lasting longer than 3 hours (Figure 6c)

\section{(a)}

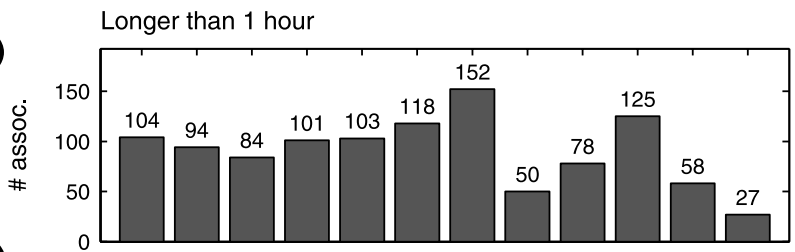

(b)

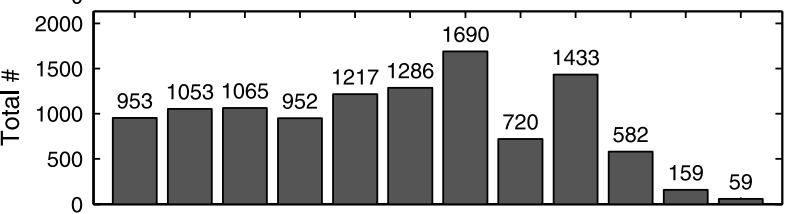

(c)

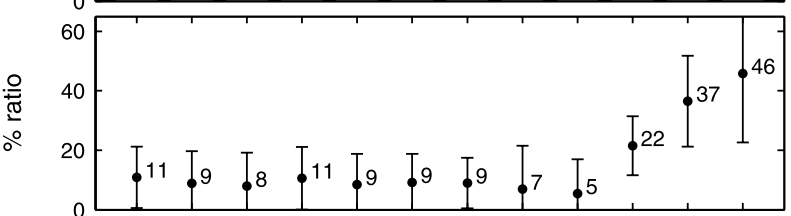

(d)

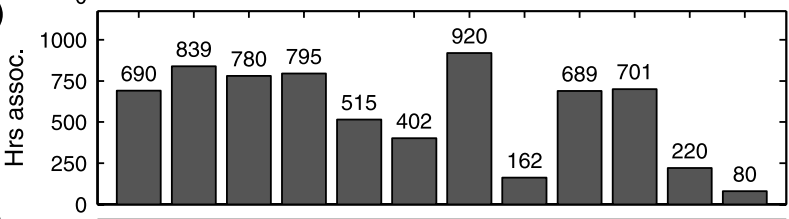

(e)

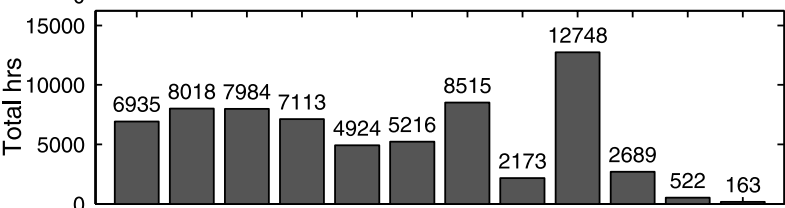

(f)

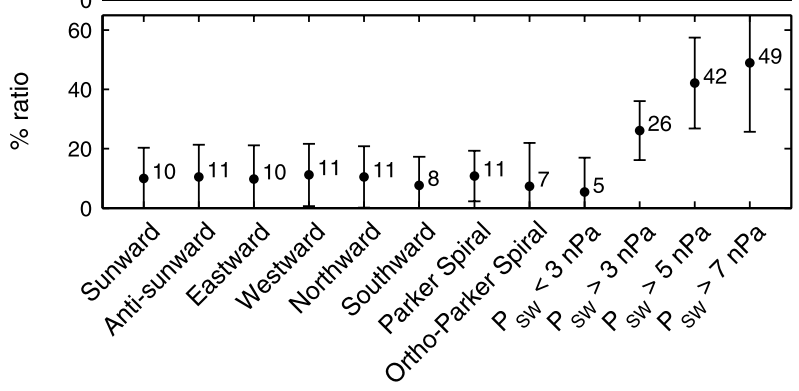

Figure 7. The coincidence of prolonged interplanetary conditions lasting longer than 1 hour, such as IMF orientation and $\mathrm{P}_{\mathrm{SW}}$, with ECR events. Shown above are (a) the number of interplanetary intervals associated with ECR events, (b) the total number of intervals, and (c) the ratio of the two previous values as a percentage, i.e., the occurrence frequency. This is also given in terms of hours in Figures $7 \mathrm{~d}, 7 \mathrm{e}$, and $7 \mathrm{f}$, respectively. Uncertainties are again estimated using Poisson counting statistics. (a)

(b)
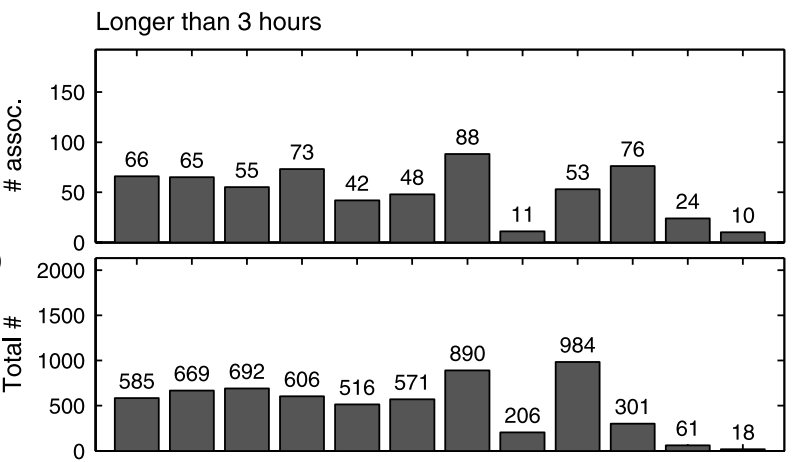

(c)

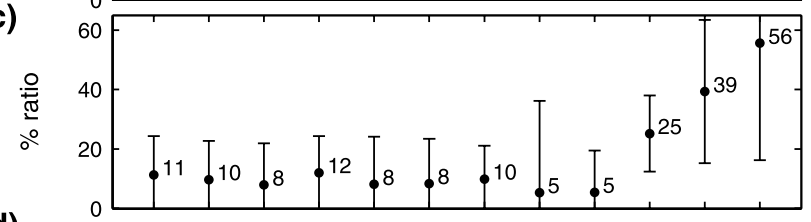

(d)

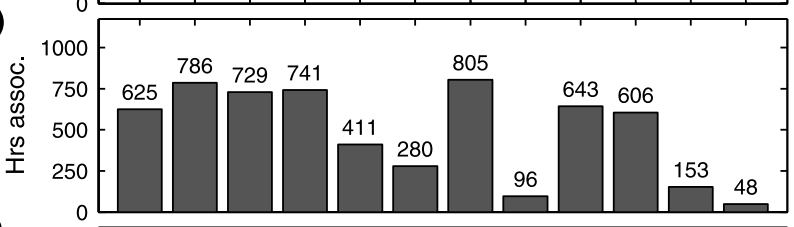

(e)

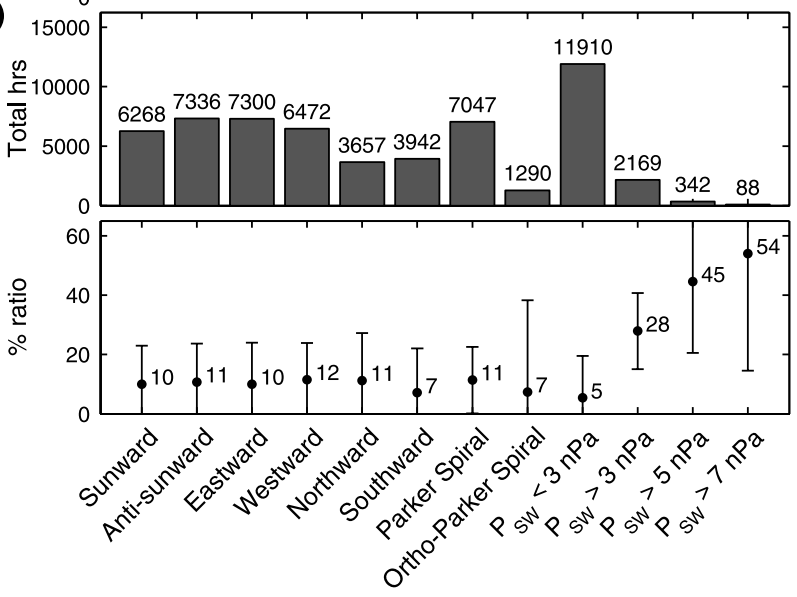

Figure 8. The coincidence of prolonged interplanetary conditions lasting longer than 3 hours with ECR events. Format same as Figure 7.

make only a small contribution to intervals lasting longer than 1 hour (Figure 6a). We note that the event of 29 May 1996 would lead us to expect ECRs to coincide with a significant number of intervals of combined strong northward IMF and high $\mathrm{P}_{\mathrm{SW}}$; as we can see in Figure 6, this is not the case. We would also expect a high coincidence with strong southward IMF and high $\mathrm{P}_{\mathrm{SW}}$; in fact, it has the worst association of any strong IMF orientation (almost $2 \sigma$ below the mean) and the largest uncertainties.

[29] For intervals lasting longer than an hour (Figure 5b), more ECRs are observed during southward IMF (59\%) than for northward IMF (48\%). For intervals of 3 hours or longer (Figure 5d) or for strong orientations lasting longer than both 1 and 3 hours (Figures $6 \mathrm{~b}$ and 6d, respectively), this relationship is reversed. Therefore it appears that the longer and more extreme the conditions, the less likely an ECR is to be associated with southward IMF compared with northward IMF. We also observe that westward IMF is preferred over eastward IMF for intervals lasting longer than 1 and 
3 hours (Figures $5 \mathrm{~b}$ and $5 \mathrm{~d}$, respectively). This is again seen in the strong orientations lasting longer than an hour (Figure $5 b$ ). However, this is not seen in events lasting longer than 3 hours, but the poor sampling means that we cannot state whether there has been a change in IMF dependence. This could be associated with the Svalgaard-Mansurov effect [Svalgaard, 1973; Mansurov, 1969], which is known to move the cusp in the east-west direction depending on the sense of

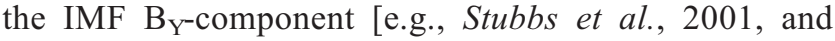
references therein]. We also note a preference for sunward IMF over antisunward IMF, particularly for intervals lasting longer than an hour (Figures $5 b$ and $6 b$ ).

\subsection{Association of Interplanetary Conditions With ECRs}

[30] In this section we approach the coincidences from the perspective of interplanetary parameters associated with

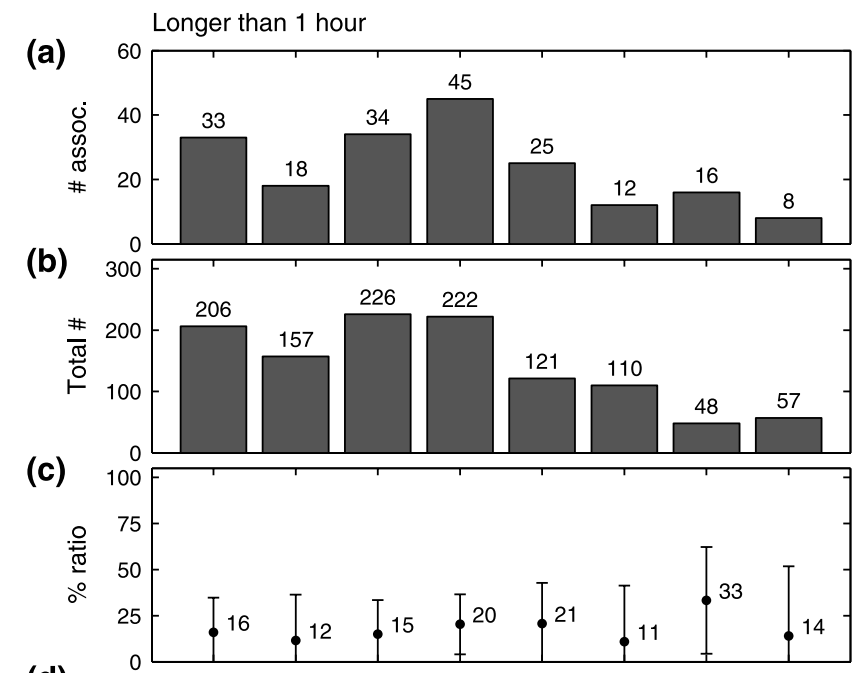

(d)

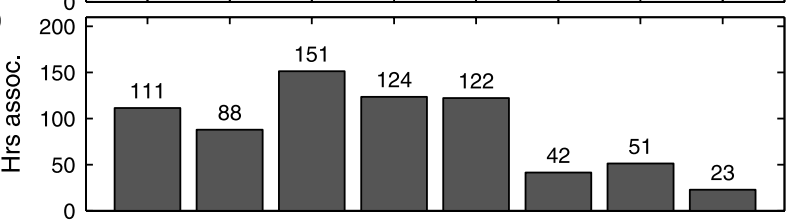

(e)

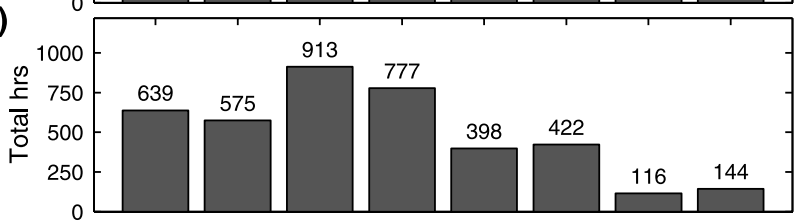

(f)

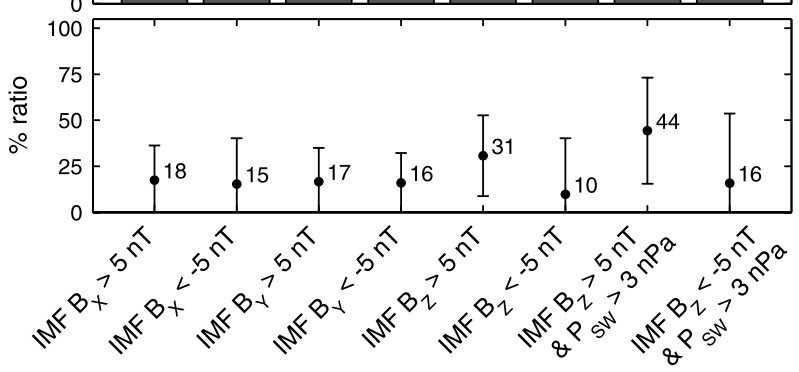

Figure 9. Same format as Figure 7; however, in this case the magnitude of the IMF component must remain above $5 \mathrm{nT}$. Also shown are the coincidences for prolonged intervals of strong magnetic magnitude $(>|5| \mathrm{nT})$ in the IMF $\mathrm{B}_{\mathrm{Z}}$-component coinciding with prolonged high $\mathrm{P}_{\mathrm{SW}}(>3 \mathrm{nPa})$.

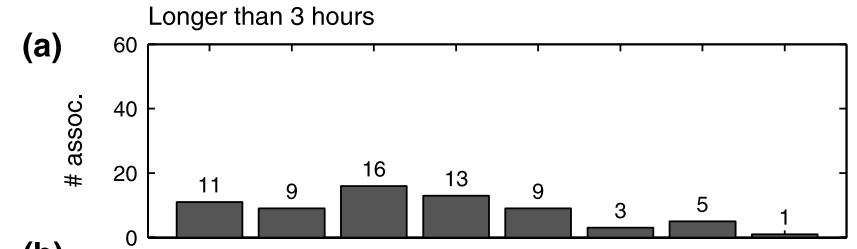

(b)

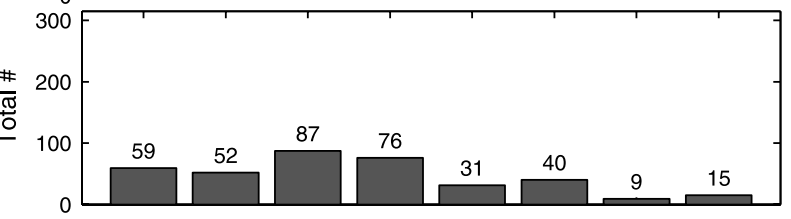

(c)

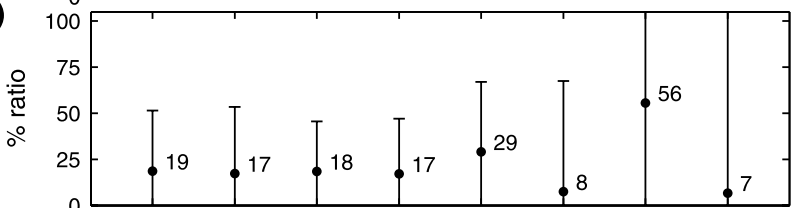

(d)

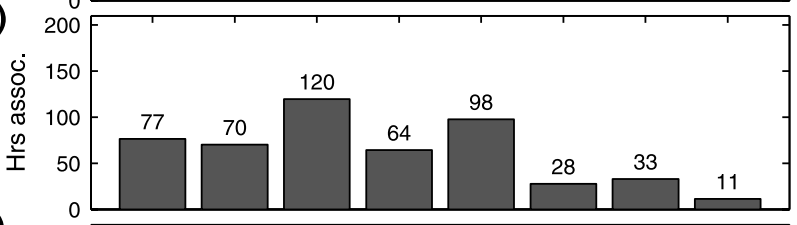

(e)

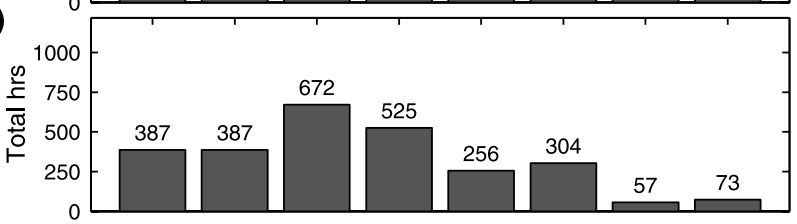

(f)

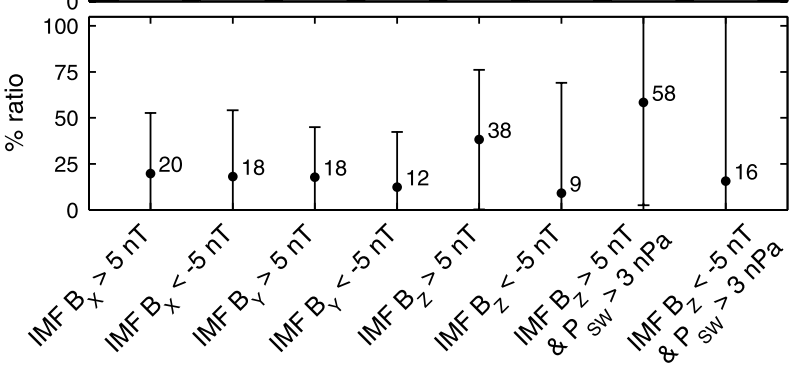

Figure 10. The coincidence of prolonged interplanetary conditions lasting longer than 3 hours with ECR events. Format same as Figure 9.

ECRs. The criteria set for overlaps between intervals in the solar wind and ECRs is the same as those discussed earlier in section 4.2. From Figures 7 and 8 we can see that no prolonged orientations of the IMF coincide very frequently with ECRs, whether the IMF orientation lasts for longer than 1 or 3 hours. The uncertainties here are also quite large relative to the observed coincidences. However, the periods of prolonged high $\mathrm{P}_{\mathrm{SW}}(>3 \mathrm{nPa},>5 \mathrm{nPa}$, and $>7 \mathrm{nPa})$ coincide with ECRs quite frequently; for periods lasting longer than 1 hour and 3 hours coincidences range from 22 to $56 \%$, as shown in Figures $7 \mathrm{c}$ and $8 \mathrm{c}$. (The occurrences for $\mathrm{P}_{\mathrm{SW}}>7 \mathrm{nPa}$ are almost $2.5 \sigma$ above the mean.) This by no means shows that intervals of prolonged strong $\mathrm{P}_{\mathrm{SW}}$ are the driver behind ECRs but does suggest they may, on occasion, play an important role. The slight preference for westward IMF over eastward IMF and sunward IMF over antisunward IMF, as discussed in section 4.2, is not seen here, thus suggesting that the previous result was probably due to orbital sampling. The intervals lasting longer than 3 hours, 
shown in Figure 8, contribute between 50 and $70 \%$ of the intervals lasting longer than an hour, shown in Figure 7, thus showing that they make a significant contribution to the total sample. Therefore we can typically expect intervals lasting longer than 3 hours, rather than shorter intervals, to coincide with ECRs.

[31] The association between prolonged strong orientation of the IMF and ECRs, shown in Figures 9 and 10, is slightly better than for the prolonged intervals, although it is still generally poor. For this sort, IMF $\mathrm{B}_{\mathrm{Z}}>5 \mathrm{nT}$ shows relatively good concurrence, particularly for periods longer than 3 hours where the coincidence is $38 \%$ (for number of hours associated with ECRs). Coincidences of periods of IMF $\mathrm{B}_{\mathrm{Z}}<-5 \mathrm{nT}$ with ECRs are consistently much lower than for other IMF orientations. As with the above results, no significant preferences are shown for IMF $\mathrm{B}_{\mathrm{X}}>5 \mathrm{nT}$ over IMF $\mathrm{B}_{\mathrm{X}}<-5 \mathrm{nT}$ or for IMF $\mathrm{B}_{\mathrm{Y}}>5 \mathrm{nT}$ over IMF $\mathrm{B}_{\mathrm{Y}}<-5 \mathrm{nT}$. From a comparison of the number of intervals associated with ECRs, we can see that the intervals of prolonged strong orientation are a small part of the total sample.

[32] The only significant association with ECRs is for prolonged intervals of IMF $\mathrm{B}_{\mathrm{Z}}>5 \mathrm{nT}$ and $\mathrm{P}_{\mathrm{SW}}>3 \mathrm{nPa}$, e.g., $58 \%$ of hours are associated with ECRs for intervals $>3$ hours, which is more than $2 \sigma$ above the mean. This suggests that when these conditions prevail an ECR is highly likely to be observed by Polar, particularly when these conditions last longer than 3 hours. However, the sampling statistics for these conditions are quite poor; therefore we need to be careful about deducing too much from this result. In contrast, the coincidence of prolonged intervals of IMF $\mathrm{B}_{\mathrm{Z}}<-5 \mathrm{nT}$ and $\mathrm{P}_{\mathrm{SW}}>3 \mathrm{nPa}$ is much lower, with only $16 \%$ of hours associated with ECRs, both for intervals $>3$ hours and intervals $>1$ hour. This suggests that highly active subsolar reconnection rarely has a significant role in creating the ECRs observed by MICS.

\section{Discussion}

\subsection{Orbital and Seasonal Variations}

[33] The change in peak occurrences and occurrence frequencies from the fall in 1996 to the spring in 1997, as shown in Figure 4, can be attributed to the precession of the Polar orbit apogee shown Figure 2a. In 1996 the apogee was oriented toward the Sun in the fall, thus making that the favorable season for ECR observation. By the following spring, apogee was nearing the ecliptic pole, showing a preference for neither season; this enabled Polar to observe ECRs around April and May. By the fall of 1997, Polar observed very few ECRs, even though apogee was in a similar location relative to the Sun as it was the previous April. The orbital plane in the fall was tilted toward dawn; however, this would not be expected to lower the occurrence of cusp observations which, on average, is roughly symmetric about noon (e.g., Newell and Meng [1992], Stubbs et al. [2001, 2004], and also seen here for ECRs in Figure 1a). In fact, these previous studies of the cusp have shown a prenoon bias, which would suggest that cusp observations would be more likely when the orbital plane is tilted toward dawn. The ability of Polar to observe ECRs through 1996 was very much dependent on the sunward tilt of orbital plane from late May through to early September of that year, as shown in Figure $2 b$.
[34] During the final 2 years, ECRs were observed mainly during the spring and summer, with the peak occurrence in May for both years. At these times, both the orbit apogee and tilt of the orbital plane of Polar were oriented toward the Sun (see Figure 2), allowing it to spend more time in the region of the midaltitude cusp, as can be seen in Figure 4d. From Figure $4 \mathrm{f}$ we can see that the occurrence frequencies in the spring of 1997 to 1999 were much greater than those in the fall of 1996 and 1997. In fact, compared with the fall of 1996, the occurrence frequencies in the subsequent spring times were between 45 and $180 \%$ higher. This suggests that there may also be a seasonal variation which encourages ECR occurrences in late spring relative to early fall. In Figure 4f, the occurrence frequencies in 1999 are lower than those in 1998; this suggests that the precession of the orbit may be moving Polar equatorward of the midaltitude cusp. However, this is not seen to the same extent in occurrence frequencies shown in Figure 4g. Also, ECR occurrences during the late spring and summer of 1996 show that although the orbit is not favorable to cusp observations at this time, they are still seen. It is possible that a seasonal variation allows Polar to observe ECRs at these times.

[35] From the comparison with the Sibeck et al. [1991] magnetopause model shown in Figure 3, we note that the orbit was moving closer to the magnetopause in 1998 and 1999. Therefore it is likely that at these times Polar was, on occasion, entering the magnetosheath during intervals of high $\mathrm{P}_{\mathrm{SW}}$, as well as observing the cusp. This could be a factor in the increased occurrence frequency of ECRs during these years.

\subsection{IMF Dependencies and Magnetopause Reconnection}

[36] The implications of the preference for sunward IMF over antisunward IMF for intervals longer than an hour (Figures $5 \mathrm{~b}$ and $6 \mathrm{~b}$ ) would depend on the sense of IMF $\mathrm{B}_{\mathrm{Z}}$. Under southward IMF conditions, we would expect a sunward IMF component to cause plasma on reconnected field lines to be accelerated away from the subsolar point more quickly toward the northern hemisphere than the southern hemisphere [e.g., Cowley et al., 1991]. This would help energize ions above the $1 \mathrm{keV}$ detection threshold of MICS. Under northward IMF conditions, a sunward IMF component would be expected to cause lobe reconnection to preferentially occur in the southern hemisphere [e.g., Cowley et al., 1991]. This could lead to open field lines being draped over the dayside magnetosphere in the region of the northern cusp. Both processes discussed above could enhance the likelihood of observing ECRs.

[37] The relatively low concurrences of prolonged intervals of IMF $B_{Z}<-5 n$ T with ECRs compared with IMF $\mathrm{B}_{\mathrm{Z}}>5 \mathrm{nT}$ (see Figures 9 and 10) could be explained by the velocity dispersion effect and the fact that MICS observes the high-energy tail of the cusp ion distribution. When magnetosheath ions enter the cusp on open field lines, the high-energy $(>1 \mathrm{keV})$ ions are observed closest to the field lines mapping to the $\mathrm{X}$-line and the low-energy $(<1 \mathrm{keV})$ ions are observed furthest from these field lines [Lockwood, 1995a]. This is the velocity dispersion effect, as described by Burch et al. [1982]. Therefore during southward IMF the high-energy ions are observed entering 
at the equatorward edge of the cusp. These ions then mirror and leave the cusp and enter the mantle in a narrow range of latitudes. As mentioned before, it is possible for Hydra to observe cusp signatures without MICS observing an ECR signature. Therefore we suggest that the Polar orbit may be traversing the poleward edge of the cusp during southward IMF, where the ions are below the energy threshold of MICS. It is possible that these conditions move the high-energy cusp ions equatorward of Polar when it is at apogee, i.e., when it is at the required altitudes to observe ECRs. This would be especially significant during 1996 and 1997 when apogee was at high latitudes (see Figures 2 and 3). Likewise, for a northward IMF cusp we would expect to see high-energy ions at the poleward edge of the cusp, closer to Polar's orbit near apogee. This effect would also be exaggerated by the fact that the cusp is located further equatorward (poleward) during southward (northward) IMF due to flux erosion from the magnetopause [Newell and Meng, 1988; Stubbs et al., 2001]. Tsyganenko and Russell [1999] also noticed that the equatorward edge of the midaltitude cusp was poorly sampled in their survey of magnetic cusp signatures, which concurs with the findings here. As suggested by Grande et al. [1997] and Russell et al. [1998], ions are more likely to be trapped in a northward IMF cusp due to the kinked magnetic field lines which produce magnetic trapping geometries. This can extend the lifetime and latitudinal extent of high-energy ions in the cusp.

[38] The results from section 4.3 suggest that ECRs are more likely during prolonged intervals of high solar wind dynamic pressure $\left(\mathrm{P}_{\mathrm{SW}}>3 \mathrm{nPa}\right)$ and prolonged strong northward IMF $\left(\mathrm{B}_{\mathrm{Z}}>5 \mathrm{nT}\right)$, especially when observed together. The ECR events coinciding with these intervals are spread throughout the 3.25 years of observation, suggesting Polar was sampling regions where this could occur at some time during each year. Northward IMF is associated with flux erosion at the tail lobes, which moves the magnetopause boundary closer to the Earth, and is consistent with the hypothesis of Grande et al. [1997]. However, this can only explain a handful of ECRs, such as the 29 May 1996 event. Overall, there is no clear association with the behavior of any one interplanetary parameter to explain all ECRs, as shown in Figures 5 and 6 , thus suggesting that they occur under any interplanetary conditions.

[39] An important point to note when interpreting these results concerns the extent of the actual cusp compared with the ECR observation region. The cusp varies in location and extent, such that at any given instant it is smaller than the ECR observation region (defined using all ECR trajectories), possibly by as much as a factor of 2 or 3 . This is supported by the occurrence frequency distributions shown by Stubbs et al. [2004] for ions observed by Polar MICS, which show that the probability of observing the cusp is at most $35 \%$. This peak occurs near local noon at $\sim 80^{\circ}$ magnetic latitude, with probabilities falling off to $10-15 \%$ within $2^{\circ}$ or $3^{\circ}$ of latitude. This result is also seen in the survey by Newell and Meng [1992], which uses data from the low-altitude DMSP spacecraft. If we had a more sophisticated method of defining the cusp extent and location, then in Figures 7-10 we would expect the number of periods of prolonged interplanetary conditions associated with the ECR observation region to be significantly reduced. This, in turn, would increase the coincidences, which would likely strengthen the result that northward IMF and high $\mathrm{P}_{\mathrm{SW}}$ are often associated with ECRs.

\subsection{Effect of Magnetopause Indentations at the Cusps}

[40] In the vicinity of the cusps it is important to consider the possible effects of an indentation in the magnetopause boundary [e.g., Mead and Beard, 1964]. This effect has recently been modeled by Boardsen et al. [2000], so in Figure 11 we have qualitatively compared their model with the ECRs to determine whether it is possible that some are traversals of the magnetopause indentation at the cusp. Boardsen et al. [2000] developed an empirical model which is dependent on solar wind dynamic pressure $\left(\mathrm{P}_{\mathrm{SW}}\right)$, dipole tilt angle $(\Psi)$, and the IMF Z-component $\left(B_{Z}\right)$. In order to improve the comparison, ECRs were divided into three roughly equal bins for both dipole tilt and IMF $B_{Z}$ for events dominated by $\Psi<5^{\circ}, 5^{\circ}<\Psi<15^{\circ}$, and $\Psi>15^{\circ}$ and $\mathrm{B}_{\mathrm{Z}}<-2.5 \mathrm{nT},-2.5 \mathrm{nT}<\mathrm{B}_{\mathrm{Z}}<1.0 \mathrm{nT}$, and $\mathrm{B}_{\mathrm{Z}}>1.0 \mathrm{nT}$, respectively. This produced nine sets of ECRs binned by $\Psi$ and IMF $\mathrm{B}_{\mathrm{Z}}$, which are plotted with the Boardsen et al. [2000] model in Figure 11. When plotting the magnetopause model with these events we used the approximate mean values of $\mathrm{P}_{\mathrm{SW}}, \Psi$, and IMF $\mathrm{B}_{\mathrm{Z}}$ coinciding with the ECR observations in each of the nine bins. Therefore we kept $\mathrm{P}_{\mathrm{SW}}=5 \mathrm{nPa}$ for all plots; for positive, near-zero, and negative values of the dipole tilt (shown from left to right) we used $\Psi=20^{\circ}, 10^{\circ}$, and $-4^{\circ}$, respectively, and for northward, near-zero, and southward IMF (shown from top to bottom), we used IMF $B_{Z}=4.0 \mathrm{nT},-0.75 \mathrm{nT}$, and $-3.5 \mathrm{nT}$, respectively. From a comparison with Figure 3 we can see that for $\mathrm{P}_{\mathrm{SW}}=5 \mathrm{nPa}$ the Sibeck et al. [1991] model would place all ECRs well within the magnetosphere.

[41] From Figure 11, it appears that some of the ECRs could have been due to a traversal of a magnetopause indentation at the cusp, particularly for ECRs when $\Psi$ was positive or near zero and accompanied by northward or near-zero IMF. This model clearly shows how the magnetopause indentation at the cusp, and therefore the cusp, moves equatorward for positive dipole tilt angles (i.e., during the summer months), as well as for IMF $\mathrm{B}_{\mathrm{Z}}<0$. This is consistent with the cusp moving equatorward of Polar during southward IMF, as argued above. We also notice that the greater the dipole tilt angle or the more northward the IMF, the greater the magnetopause indentation at the cusp. This is all consistent with the high ECR occurrences observed during late spring and early summer when $\Psi$ is approaching its maximum, as well as the relatively high coincidence with intervals of prolonged northward IMF. With this seasonal variation in the location of the magnetopause indentation at the cusp, we might expect the ECRs to peak in June, as it is the month of maximum $\Psi$. However, from Figure 4d, we can see that from 1997 to 1999 Polar spends most time in the cusp region around May; therefore the orbit appears to be responsible for the decrease in ECR occurrences after this month. Sotirelis and Meng [1999] developed a pressure balance model using the T96 model, which typically shows an even greater magnetopause indentation at the cusps than shown here. The ECRs in Figure 11 not falling within the magnetopause indentation 


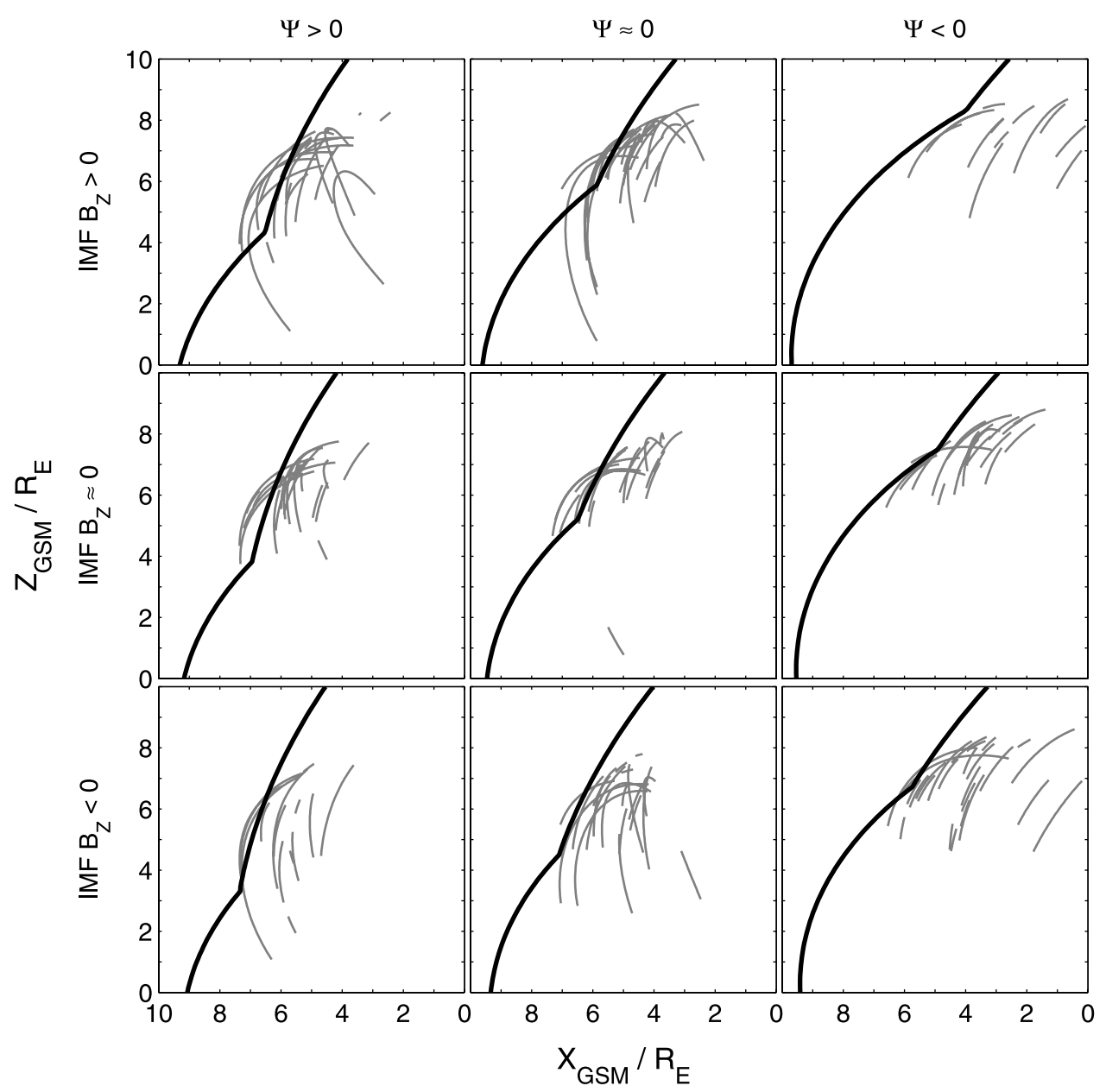

Figure 11. Comparison of ECRs with the Boardsen et al. [2000] magnetopause model, plotted as a function of $\mathrm{X}$ and $\mathrm{Z}$ in GSM coordinates. This empirical model of the magnetopause includes indentations at the cusps and is a function of solar wind dynamic pressure $\left(\mathrm{P}_{\mathrm{SW}}\right)$, dipole tilt angle $(\Psi)$, and the IMF Z-component (IMF $B_{Z}$ ). In this figure we have binned the ECRs by $\Psi$ and IMF $B_{Z}$. When plotting the magnetopause model, we used the approximate mean values of $P_{S W}, \Psi$, and IMF $B_{Z}$ coinciding with ECR observations. Therefore we kept $\mathrm{P}_{\mathrm{SW}}=5 \mathrm{nPa}$ for all plots; for $\Psi>0, \Psi \approx 0$, and $\Psi<0$ (from left to right), we use $\Psi=20^{\circ}, 10^{\circ}$, and $-4^{\circ}$, respectively, and for $I M F B_{Z}>0, I M F B_{Z} \approx 0$, and IMF $B_{Z}<0$ (from top to bottom), we use IMF $B_{Z}=4.0 \mathrm{nT},-0.75 \mathrm{nT}$, and $-3.5 \mathrm{nT}$, respectively.

in the cusp could have either coincided with higher $\mathrm{P}_{\mathrm{SW}}$ or been on open field lines in the cusp or in the magnetosheath. However, it should be remembered that this is not a rigorous comparison and there are other factors to consider, such as the fact that in an empirical model the magnetopause shape is smoothed in time and space.

[42] From our data it is not possible to absolutely distinguish any seasonal variation; this is due to the cusp not being sampled sufficiently throughout the year. However, they can be inferred from the occurrence distribution over a period of several years when the orbit precesses across the ecliptic pole, as is the case here (see Figure 2a).

\subsection{Comparisons With Other Studies}

[43] It is also interesting to compare our results with the study of cusp energetic particle (CEP) events by Chen et al. [1998]. Seventy-five individual CEP events were identified on 33 separate orbits during 1996. During a CEP event the intensity of helium ion fluxes observed by MICS is anti- correlated with the local magnetic field magnitude but correlated with turbulent magnetic energy density. These events are also associated with an enhancement of ULF magnetic noise $(\sim 1$ to $100 \mathrm{~Hz})$ and $\mathrm{MeV}$ helium ions. As with the ECR events, the helium and oxygen ions in CEP events have high charge states consistent with a solar wind source. The definition of a CEP event is similar in many respects to that of an ECR but more restrictive. Indeed in 1996, 24 out of 33 ECRs are associated with CEP events (73\%), and 23 out of 33 CEP events are associated with ECRs $(70 \%)$, based on a comparison with the list of Chen et al. [1998]. More recently, the term cusp diamagnetic cavity (CDC) has been used to refer to ECR-type events [e.g., Fritz et al., 2003]. If a CDC contains appreciable fluxes of 10$100 \mathrm{keV}$ particles, then it is also a CEP event.

[44] It has been proposed that these CEP events are produced by a local acceleration mechanism associated with the high-altitude dayside cusp that energizes the low-energy helium from the magnetosheath [e.g., Chen et al., 1998; 
Chen and Fritz, 1998; Fritz et al., 2003]. Chen and Fritz suggested that ions could be trapped in the high-altitude cusp if it formed a quadrupole magnetic field structure where the adiabatic invariants of the particles could be conserved, as suggested by Sheldon et al. [1998]. They argued that this would allow inductive electric fields produced by the ULF wave turbulence to resonantly accelerate the ions. Recent observations by Cluster II have shown that the cusp is extremely dynamic on short time scales [e.g., Cargill et al., 2001], so the assumption that the dynamic behavior of the cusp is approximately static on the time scales of the ion motion appears to be invalid [Stubbs, 2002; Fritz et al., 2003]. This led Fritz et al. [2003] to suggest that there must be some temporary trapping mechanism in the cusp.

[45] On the other hand, Chang et al. [1998] and Trattner et al. [2001] have argued that the energetic $\mathrm{He}^{2+}$ ions have been accelerated at a quasi-parallel bow shock, subsequently convected downstream, and then entered the cusp along open field lines. They argued that the shocked magnetosheath plasma downstream of a quasi-parallel bow shock during these events consisted mainly of a directly transmitted population $\left(<2 \mathrm{keV} \mathrm{e}^{-1}\right)$ and ions initially reflected at the shock and subsequently convected downstream $\left(<10 \mathrm{keV} \mathrm{e}^{-1}\right)$. This corresponds to the population we have defined as ECRs, when observed for prolonged periods by MICS. The energetic ion distribution consists of a bow shock-accelerated diffuse ion component from 10 to $150 \mathrm{keV} \mathrm{e}^{-1}$ [Fuselier, 1994; Trattner et al., 1994] and a high-energy component above $150 \mathrm{keV} \mathrm{e}^{-1}$ of magnetospheric origin [e.g., Scholer et al., 1981]. Both sets of studies used CEP events from 1996, which were also identified as ECRs.

[46] Chen et al. [1998] performed a similar analysis of CEP occurrences to that described in section 4.1 for the events they identified in 1996. They concluded that the peak they observed in September 1996, also seen in Figure 4, was due to a seasonal rather than an orbital variation, as the distribution retained the same form after normalization. We have shown that this is not the case, as in subsequent years occurrences are enhanced around late spring and early summer and disappear during the fall. This strongly suggests that the evolution in the orbit dictates the time of year when ECR and CEP events are best observed, as well as there being a seasonal variation.

\section{Summary and Conclusions}

[47] This paper has addressed the statistical properties of extended cusp-like regions and their relationship with the Polar orbit, seasonal variations, and interplanetary conditions. The major results are as follows:

[48] 1. ECRs are only observed when Polar is in the dayside magnetosphere around noon. The time of year at which this occurs is dependent on the orbit apogee and inclination (see Figure 2). The precession of the orbit, which leads to a variation of the latitude of apogee each year, explains both the peak occurrence originally around September and October 1996 and the subsequent shifts to around April and May (see Figure 4). The ECR occurrences during the periods of spring and summer sampling were consistently higher than the occurrences during the fall, suggesting that there is a seasonal dependence. This is demonstrated by the higher occurrences in spring 1997 than in the fall of 1997, when the orbits were in roughly the same location relative to the Sun. The $\mathrm{P}_{\mathrm{Sw}}$-dependent magnetopause model of Sibeck et al. [1991] predicts that the vast majority of ECRs should remain inside the magnetopause for $\mathrm{P}_{\mathrm{SW}}$ up to $7.0 \mathrm{nPa}$, as shown by Figure 3 .

[49] 2. Overall, ECRs as defined here do not coincide preferentially with any prolonged orientation of the IMF or high $\mathrm{P}_{\mathrm{SW}}$, thus showing that they occur under all interplanetary conditions (see Figures 5 and 6). The distribution of prolonged intervals of interplanetary conditions observed during ECRs simply mirrors the average distributions expected in the solar wind [e.g., Hapgood et al., 1991]. For prolonged intervals of strong orientations of the IMF the coincidences worsen, as expected for fewer interplanetary intervals, with IMF $\mathrm{B}_{\mathrm{Z}}<-5 \mathrm{nT}$ having the worst association.

[50] 3. Infrequent solar wind conditions, in particular a prolonged interval of both strong northward IMF $\left(\mathrm{B}_{Z}>\right.$ $5 \mathrm{nT})$ and high dynamic pressure $\left(\mathrm{P}_{\mathrm{SW}}>3 \mathrm{nPa}\right)$, are often accompanied by an ECR (see Figure 7 to 10). However, when this condition lasts for an hour or longer it only accounts for 16 out of 184 ECRs ( 9\%). ECRs are more prevalent during prolonged high $\mathrm{P}_{\mathrm{SW}}$, but this is also not a common cause. This shows that some conditions encourage the formation of ECRs. It is also important to remember that the ECR observation region defined here is a large static region, unlike the actual cusp which is more dynamic. If we were able to fully take this into account, the concurrence frequencies would be significantly higher.

[51] 4. The concurrence frequency is significantly less for intervals of IMF $B_{Z}<-5 \mathrm{nT}$ than for intervals of IMF $B_{Z}>$ $5 \mathrm{nT}$. This is most likely because during these intervals of southward IMF, Polar is in the poleward edge of the cusp where ions are below the $1 \mathrm{keV}$ energy threshold of MICS. As MICS detects the high-energy ion component of the cusp, it preferentially observes ions on open field lines nearest to the X-line (i.e., at the equatorward edge of the cusp for southward IMF and the poleward edge for northward IMF). The effect would be exaggerated with decreasing IMF $\mathrm{B}_{\mathrm{Z}}$ as the cusp moved equatorward and by the acceleration away from a subsolar X-line being greater than from an X-line in the tail lobe, which likely results in the cusp being narrower for southward IMF. Also, the greater possibility for magnetic trapping geometries in a northward IMF cusp would increase both the latitudinal extent and trapping lifetime of the ions in the cusp.

[52] 5. The Boardsen et al. [2000] model shows that the magnetopause indentation at the cusp is largest for positive dipole tilt angle and northward IMF (see Figure 11). Comparisons show that some ECRs are traversals of this indentation region, which contributes to the higher ECR occurrences around late spring, and the high concurrence frequencies for northward IMF. This is also consistent with magnetic field observations [Tsyganenko and Russell, 1999]. The inferred seasonal variation explains why there is no significant association with any particular set of interplanetary conditions.

[53] So what are ECRs? They appear to have three distinct causes and so can relate to extended intervals in (1) the cusp on open field lines, (2) the magnetosheath, and (3) the magnetopause indentation at the cusp. The latter 
would have the effect of widening the cusp such that it covers a greater range of invariant latitudes and local times. Most importantly, it would allow magnetosheath plasma to approach closer to the Earth in the region of the cusps without having to enter the magnetosphere, as has been observed.

[54] In this paper we have worked toward an understanding of how extended cusp-like regions at midaltitudes are formed by investigating their dependence on seasonal variations and interplanetary conditions. This will also help to better understand the source of the energetic $(>10 \mathrm{keV})$ particles therein, whether they are energized in the cusp [e.g., Fritz at al., 2003] or at the quasi-parallel bow shock [e.g., Trattner et al., 2001].

\section{Appendix A: Estimating Solar Wind Conditions at the Earth}

[55] For this study we require a reliable and consistent method for estimating the time delay between observations of the interplanetary magnetic field and plasma and its arrival at the Earth. Using Alfvén's frozen flux theorem, we can assume that the IMF $\left(\mathbf{B}_{\mathrm{IMF}}\right)$ is convected at the solar velocity $\left(\mathbf{V}_{\mathrm{SW}}\right)$, allowing us to calculate a reasonable estimate for the propagation time to the Earth. (However, on rare occasions when both the solar wind flow and the IMF are radial, such that $\mathbf{V}_{\mathrm{SW}} \times \mathbf{B}_{\mathrm{IMF}}=0$, particles simply stream along the field lines.) In the solar wind the radial flow component is dominant, such that the bulk of the motion is along the Sun-Earth line (GSE X-axis), i.e., $\mathbf{V}_{\mathrm{SW}} \approx \mathrm{V}_{\mathrm{X}}$, where $\mathrm{V}_{\mathrm{X}}$ is the flow velocity in the GSE X direction. If we observe the solar wind at a point along the Sun-Earth line, the delay time to the center of the Earth is

$$
\Delta t=\frac{X}{V_{X}},
$$

where $\mathrm{X}$ is the distance from the spacecraft to the center of the Earth in the GSE X direction. We use equation (A1) to estimate the delay time to the Earth under the assumption that the structure and properties of the solar wind do not vary greatly over distances of $\sim 100 \mathrm{R}_{\mathrm{E}}$ transverse to the Sun-Earth line, as is appropriate for well-organized events.

[56] In calculating the time to the center of the Earth, we assume that the time taken for the shocked solar wind plasma to travel through the subsolar magnetosheath and reach the magnetopause is approximately equal to $\mathrm{X}_{\mathrm{BS}} / \mathrm{V}_{\mathrm{SW}}$, where $\mathrm{X}_{\mathrm{BS}}$ is the geocentric standoff distance to the bow shock. Alternatively, we could use estimates based on magnetosheath flow models and estimates of plasma propagation times to the cusps [e.g., Spreiter et al., 1966]. However, these methods all involve errors and probably add little to the accuracy of the delay estimate.

[57] The solar wind plasma data were used to estimate the delay time from the spacecraft in interplanetary space to the Earth, as discussed in section 2 for Wind and ACE, and then joined with the magnetic field data and averaged into 2-min bins. Although the final bin width is $2 \mathrm{~min}$, the value given to any parameter is an average over $5 \mathrm{~min}$ around the bin midpoint. We have done this because there is at least a 5-min uncertainty in the propagation time. Also, the $\mathrm{V}_{\mathrm{SW}}$ and positions used in the calculation of the propagation time are averaged over $20 \mathrm{~min}$ around the midpoint, in an attempt to smooth out wave activity and resolve the velocity of the underlying large-scale structures propagating through the solar wind.

[58] Wind frequently traversed the bow shock; therefore we needed to remove these times from the data set to ensure we did not sample shocked magnetosheath plasma. We imposed a condition that if the position of Wind in the GSE X direction were less than $20 \mathrm{R}_{\mathrm{E}}$, the code would check to see if it was located within the bow shock, as defined by the Howe and Binsack [1972] model:

$$
S_{B S}=H\left[\left(\frac{D+S-X}{D}\right)^{2}-1\right]^{1 / 2} .
$$

In equation (A2), $\mathrm{S}_{\mathrm{BS}}$ is the distance to the bow shock in the GSE $\left(\mathrm{Y}^{2}+\mathrm{Z}^{2}\right)^{1 / 2}$ direction for a given position in the GSE $\mathrm{X}$ direction; $\mathrm{H}=56.7$ and $\mathrm{D}=177.7$ and are both constants and $\mathrm{S}$ is the distance to the subsolar bow shock. All distances are in units of $R_{E}$. Howe and Binsack originally set $\mathrm{S}=13.5 \mathrm{R}_{\mathrm{E}}$; this is quite close to the Earth for most interplanetary conditions. Therefore we vary this distance using the Sibeck et al. [1991] magnetopause model prediction for the distance to the subsolar magnetopause, $\mathrm{S}_{\mathrm{MP}}$, as discussed later. To obtain a value for $\mathrm{S}$ from $\mathrm{S}_{\mathrm{MP}}$, we used the empirical relationship $\mathrm{S} / \mathrm{S}_{\mathrm{MP}}=1.3$ from Fairfield [1971]. As an additional safety factor, we added a $20 \%$ uncertainty margin to the result from equation (A2) to ensure we did not use data within the bow shock. From the data used by Howe and Binsack, this additional factor should encompass all the data used to model the bow shock boundary. By specifically removing Wind observations made within the bow shock, it allows us to use the remaining data taken around the bow shock, which can include data taken downstream of the Earth.

[59] As mentioned above, the Sibeck et al. [1991] solar wind dynamic pressure $\left(\mathrm{P}_{\mathrm{SW}}\right)$ dependent magnetopause model used to calculate the distance to the subsolar magnetopause is given by

$$
S_{M P}=\frac{-B+\sqrt{B^{2}-4 A C}}{2 A}
$$

where $\mathrm{A}=0.14, \mathrm{~B}=18.2\left(2.04 / \mathrm{P}_{\mathrm{SW}}\right)^{1 / 6}$, and $\mathrm{C}=-217.2$ $\left(2.04 / \mathrm{P}_{\mathrm{SW}}\right)^{1 / 3}$, where $\mathrm{P}_{\mathrm{SW}}$ has units of $\mathrm{nPa}$. Once we had calculated the propagation time to the Earth in GSE coordinates, we converted the magnetic field to GSM coordinates using the estimated time of arrival at the Earth. This can be important as GSM is a time-dependent coordinate system, where differences in the time of coordinate transformation of around $30 \mathrm{~min}$ or more can lead to noticeable differences in the resulting GSM parameters.

[60] Occasionally, a series of very large spikes would appear in the Wind and ACE data, which could last for days; this was particularly a problem for data from the Wind SWE instrument. As a result, we had to develop a method for filtering out this erroneous data, as it not only seriously affected the output solar wind parameters but also distorted the solar wind propagation time estimate. In processing the data to remove these spikes, we were extremely careful to remove only the erroneous data and leave any "real" data that showed a genuine sharp increase in value. 
Table A1. Values Used in Filtering Out the Spikes in the Magnetic Field and Solar Wind Plasma Data

\begin{tabular}{|c|c|c|}
\hline Filtering Parameter & Symbol & Value Used \\
\hline $\begin{array}{l}\text { Number of standard deviations from the mean } \\
\text { Maximum value for nonradial flow, }\left(\mathrm{V}_{\mathrm{Y}}{ }^{2}+\mathrm{V}_{\mathrm{Z}}{ }^{2}\right)^{1 / 2} \\
\text { Acceptable value for number density, } \mathrm{n} \\
\text { Maximum expected value for number density, } \mathrm{n} \\
\text { Maximum expected value for radial flow, } \mathrm{V}_{\mathrm{X}} \\
\text { Acceptable value for B-field magnitude, }|\mathbf{B}| \\
\text { Maximum expected value for B-field magnitude, }|\mathbf{B}|\end{array}$ & $\begin{array}{c}\mathrm{g}_{\sigma} \\
\mathrm{V}_{\mathrm{YZ} \text { MAX }} \\
\mathrm{n}_{\mathrm{a}} \\
\mathrm{n}_{\mathrm{MAX}} \\
\mathrm{V}_{\mathrm{X} \text { MAX }} \\
|\mathbf{B}|_{\mathrm{a}} \\
|\mathbf{B}|_{\text {MAX }}\end{array}$ & $\begin{array}{c}3 \\
200 \mathrm{~km} \mathrm{~s}^{-1} \\
6 \mathrm{~cm}^{-3} \\
40 \mathrm{~cm}^{-3} \\
-100 \mathrm{~km} \mathrm{~s}^{-1} \\
6 \mathrm{nT} \\
50 \mathrm{nT}\end{array}$ \\
\hline
\end{tabular}

[61] The method described here for removing erroneous data was based on a few simple assumptions, which were then applied and tested over a range of solar wind conditions and shown to work extremely well. The erroneous data was usually at least one or two orders of magnitude above the real data, allowing us to do some systematic filtering. The parameters used in the filtering process are given in Table A1.

[62] The first parameter examined was the solar wind flow perpendicular to the radial direction, $\left(\mathrm{V}_{\mathrm{Y}}^{2}+\mathrm{V}_{\mathrm{Z}}^{2}\right)^{1 / 2}$, hereafter referred to as $\mathrm{V}_{\mathrm{YZ}}$. As mentioned previously, most of the solar wind flow away from the Sun is in the radial direction, such that $\mathrm{V}_{\mathrm{SW}} \approx \mathrm{V}_{\mathrm{X}}$, so any nonradial flow is very small. From examination of a large amount of solar wind data, we found that $V_{Y Z}$ never genuinely exceeded $150 \mathrm{~km} \mathrm{~s}^{-1}$, so we set the maximum value of $\mathrm{V}_{\mathrm{YZ}}\left(\mathrm{V}_{\mathrm{YZ} \text { MAX }}\right)$ to be $200 \mathrm{~km} \mathrm{~s}^{-1}$. When $V_{Y Z}$ exceeded this value, we removed all solar wind plasma data for this time. As all moments of the solar wind plasma data used here are calculated from the same distribution function, we must assume that if one moment is erroneous then they all must be.

[63] The next parameter examined was the number density, $\mathrm{n}$. The technique used here for filtering erroneous data is slightly more sophisticated. We take a 20-min sliding window of data and increment it by $2 \mathrm{~min}$ through the entire interval of data being examined. The mean, $\langle\mathrm{n}\rangle$, and standard deviation, $\sigma_{\mathrm{n}}$, of $\mathrm{n}$ in this 20 -min window is calculated and used to determine an upper limit, $b_{U}=$ $\langle\mathrm{n}\rangle+\mathrm{g}_{\sigma} \sigma_{\mathrm{n}}$, where $\mathrm{g}_{\sigma}$ is the number of standard deviations away from the mean $\left(\langle\mathrm{n}\rangle\right.$ and $\sigma_{\mathrm{n}}$ must be calculated using at least two data points), If any of the data points exceed $b_{U}$ or $\mathrm{n}_{\text {MAX }}$ (the maximum expected value for $\mathrm{n}$ ), they are flagged as needing to be checked again. For each flagged data point, we then check to see if it exceeds what we have determined to be an "acceptable" value for $n, n_{a}$. If $n_{a}$ is not exceeded, the data point is considered to be good. This is done to ensure that we do not remove genuine "spikiness" in the data, and any missed erroneous data is not large enough to significantly distort the output parameters. If the flagged data does exceed $n_{a}$, then we take 20 min of data around each flagged data point and calculate an upper limit as before, $b_{\mathrm{U} 2}$, and then remove the data points that exceed $b_{\mathrm{U} 2}$ from this part of the calculation. From the remaining data points we calculate a further upper limit, $b_{U 3}$, if the data originally flagged as exceeding $b_{U}$ also exceeds $b_{U 3}$ then it is removed. Again, all solar wind plasma parameters calculated for this time are removed. This process is repeated through the entire interval of data being examined.

[64] Finally, with most of the erroneous solar wind plasma data having been removed, we look at the $\mathrm{V}_{\mathrm{X}}$ moments. The filtering method used is the same as that described above for the number density, except that we do not use an "accept- able" value in this calculation like the $\mathrm{n}_{\mathrm{a}}$ parameter used above, i.e., every flagged data point is rechecked. The maximum value for $\mathrm{V}_{\mathrm{X}}$ is given to be $-100 \mathrm{~km} \mathrm{~s}^{-1}$. (Note that data exceeding the maximum value is not removed, it is just flagged to be checked against data surrounding it; however, it may be removed at a later stage.) As before, if an erroneous value of $\mathrm{V}_{\mathrm{X}}$ is identified, all solar wind plasma parameters are removed at that time. This completes the removal of erroneous solar wind plasma data.

[65] The magnetic field data is checked using the magnitude of the magnetic field, $|\mathbf{B}|$. The method used here is identical to that used for the number density, as described above. The acceptable $|\mathbf{B}|$ value, $|\mathbf{B}|_{\mathrm{a}}$, is assumed to be $6 \mathrm{nT}$ and the maximum expected $|\mathbf{B}|$ value, $|\mathbf{B}|_{\text {MAX }}$, is given to be $50 \mathrm{nT}$.

[66] Assuming that the solar wind always propagates toward the Earth in a plane perpendicular to the Sun-Earth line is, in reality, not always appropriate. However, for several years worth of continuous solar wind data, as is used in statistical surveys such as this, it is currently the most reliable method for an automated calculation. By trying to take into account the orientation of the plane of the solar wind propagation in an automated routine, you risk generating significantly distorted data depending on the orientation of the IMF. Much work is being undertaken to improve estimates of the time taken for solar wind observed upstream to reach the Earth [e.g., Horbury et al., 2001]. The solar wind propagation delay code described in this paper is available on request and can be easily altered to use an alternative method for calculating the delay time.

[67] Acknowledgments. This work was funded by PPARC in the U.K. T. J. Stubbs was also funded by a National Research Council award while working at NASA/GSFC. We thank the many individuals who have made the CAMMICE program (PI T. A. Fritz) a success. We benefited from the use of Wind MFI and SWE data (PIs R. P. Lepping and K. W. Ogilvie, respectively), ACE MAG and SWEPAM data (PIs C. F. Smith and D. J. McComas, respectively), as well as Polar MFE data (PI C. T. Russell), all of which were obtained from CDAWeb at NASA/GSFC. Thanks also to M. Hapgood for advice on bow shock models.

[68] Arthur Richmond thanks Theodore Fritz and R. B. Sheldon for their assistance in evaluating this paper.

\section{References}

Acuña, M. H., K. W. Ogilvie, D. N. Baker, S. A. Curtis, D. H. Fairfield, and W. H. Mish (1995), The global geospace science program and its investigations, Space Sci. Rev., 71, 5-21.

Boardsen, S. A., T. E. Eastman, T. Sotirelis, and J. L. Green (2000), An empirical model of the high-latitude magnetopause, J. Geophys. Res., 105, 23,193-23,219.

Burch, J. L., P. H. Reiff, R. A. Heelis, J. D. Winningham, W. B. Hanson, C. Gurgiolo, J. D. Menietti, R. A. Hoffman, and J. N. Barfield (1982), Plasma injection and transport in the mid-altitude polar cusp, Geophys. Res. Lett., 9, 921-924.

Cargill, P. J., et al. (2001), First Cluster-II results of the magnetic field structure of the medium and high-altitude cusps, Ann. Geophys., 19, $1533-1544$ 
Chandler, M. O., S. A. Fuselier, M. Lockwood, and T. E. Moore (1999), Evidence of component magnetic merging equatorward of the cusp, J. Geophys. Res., 104, 22,623-22,633.

Chang, S. W., et al. (1998), Cusp energetic ions: A bow shock source, Geophys. Res. Lett., 25, 3729-3732.

Chen, J. S., and T. A. Fritz (1998), Correlation of cusp MeV helium with turbulent ULF power spectra and its implications, Geophys. Res. Lett., $25,4113-4116$

Chen, J., T. A. Fritz, R. B. Sheldon, H. E. Spence, W. N. Spjeldvik, J. F. Fennell, and S. Livi (1997), A new, temporarily confined population in the polar cap during the August 27, 1996 geomagnetic field distortion period, Geophys. Res. Lett., 24, 1447-1450.

Chen, J., T. A. Fritz, R. B. Sheldon, H. E. Spence, W. N. Spjeldvik, J. F. Fennell, S. Livi, C. T. Russell, J. S. Pickett, and D. A. Gurnett (1998), Cusp energetic particle events: Implications for a major acceleration region of the magnetosphere, J. Geophys. Res., 103, 69-78.

Cowley, S. W. H., J. P. Morelli, and M. Lockwood (1991), Dependence of convective flows and particle-precipitation in the high-latitude dayside ionosphere on the $\mathrm{X}$ and $\mathrm{Y}$ components of the interplanetary magnetic field, J. Geophys. Res, 96, 5557-5564

Fairfield, D. H. (1971), Average and unusual shock locations of the Earth's magnetopause and bow shock, J. Geophys. Res., 76, 6700.

Farrell, W. M., and J. A. Van Allen (1990), Observations of the Earth's polar cleft at large radial distances with the Hawkeye-1 magnetometer, J. Geophys. Res., 95, 20,945-20,958.

Fritz, T. A., I. A. Daglis, S. Livi, H. Sommer, B. Wilken, M. Grande, D. D. Hall, J. B. Blake, J. F. Fennell, and R. Koga (1997), The POLAR CAMMICE investigation, Tech. Rep., Cent. for Space Phys., Boston, Mass.

Fritz, T. A., J. Chen, and G. L. Siscoe (2003), Energetic ions, large diamagnetic cavities, and Chapman-Ferraro cusp, J. Geophys. Res., 108(A1), 1028, doi:10.1029/2002JA009476.

Fuselier, S. A. (1994), A comparison of energetic ions in the plasma depletion layer and the quasi-parallel magnetosheath, J. Geophys. Res., 99, 5855-5868

Grande, M., J. Fennell, S. Livi, B. Kellett, C. Perry, P. Anderson, J. Roeder, H. Spence, T. Fritz, and B. Wilken (1997), First Polar and 1995-034 observations of the midaltitude cusp during a persistent northward IMF condition, Geophys. Res. Lett., 24, 1475-1478.

Hapgood, M. A., Tulunay, M. Lockwood, G. Bowe, and D. M. Willis (1991), Variability of the interplanetary medium at 1 A.U. over 24 years: 1963-1986, Planet. Space Sci., 39, 411-423.

Horbury, T. S., D. Burgess, M. Fränz, and C. J. Owen (2001), Prediction of Earth arrival times of interplanetary southward magnetic field turnings, J. Geophys. Res., 106, 30,001-30,010.

Howe, H. C., and J. H. Binsack (1972), Explorer 33 and 35 plasma observations of magnetosheath flow, J. Geophys. Res., 77, 3334

Lepping, R. P., et al. (1995), The Wind magnetic field investigation, Space Sci. Rev., 71, 207-229.

Lockwood, M. (1995a), Location and characteristics of the reconnection X line deduced from the low-altitude satellite and ground-based observations: 1. Theory, J. Geophys. Res., 100, 21,791-21,802.

Lockwood, M. (1995b), Overlapping cusp ion injections: An explanation invoking magnetopause reconnection, Geophys. Res. Lett., 22, $1141-$ 1144.

Lockwood, M. (1997), Energy and pitch angle dispersions of LLBL/cusp ions seen at middle altitudes: Predictions by the open magnetosphere model, Ann. Geophys., 15, 1501-1514.

Lockwood, M., and M. F. Smith (1992), The variation of reconnection rate at the dayside magnetopause and cusp ion precipitation, J. Geophys. Res., 97, $14,841-14,847$.

Lockwood, M., S. E. Milan, T. Onsager, C. H. Perry, J. A. Scudder, C. T. Russell, and M. Brittnacher (2001), Cusp ion steps, field-aligned currents and poleward-moving auroral forms, J. Geophys. Res., 106, 29,55529,569

Mansurov, S. M. (1969), New evidence of a relationship between magnetic fields in space and on Earth, Geomagn. Aeron. USSR, 9, 622-623.

McComas, D. J., S. J. Bame, P. Barker, W. C. Feldman, J. L. Phillips, P. Riley, and J. W. Griffee (1998), Solar Wind Electron Proton Alpha Monitor (SWEPAM) for the Advanced Composition Explorer, Space Sci. Rev., 86, 563-612.

Mead, G. D., and D. B. Beard (1964), Shape of the geomagnetic field solar wind boundary, J. Geophys. Res., 69, 1169.

Newell, P. T., and C.-I. Meng (1988), The cusp and the cleft/LLBL: Low altitude identification and statistical local time variation, J. Geophys. Res., 93, 14,549-14,556.

Newell, P. T., and C.-I. Meng (1992), Mapping the dayside ionosphere to the magnetosphere according to particle precipitation characteristics, Geophys. Res. Lett., 19, 609-612.

Ogilvie, K. W., et al. (1995), SWE, A comprehensive plasma instrument for the Wind spacecraft, Space Sci. Rev., 71, 55-77.
Onsager, T. G., J. D. Scudder, M. Lockwood, and C. T. Russell (2001), Reconnection at the high-latitude magnetopause during northward interplanetary magnetic field conditions, J. Geophys. Res., 106, 25,46725,488 .

Paschmann, G., W. Baumjohann, N. Schopke, and T.-D. Phan (1993), Structure of the dayside magnetopause for low magnetic shear, J. Geophys. Res., 98, 13,409.

Russell, C. T., R. C. Snare, J. D. Means, D. Pierce, D. Dearborn, M. Larson, G. Barr, and G. Le (1995), The GGS/POLAR magnetic fields investigation, Space Sci. Rev., 71, 563-582.

Russell, C. T., J. A. Fedder, S. P. Slinker, X.-W. Zhou, G. Le, J. G. Luhmann, F. R. Fenrich, M. O. Chandler, T. E. Moore, and S. A. Fuselier (1998), Entry of the POLAR spacecraft into the polar cusp under northward IMF conditions, Geophys. Res. Lett., 25, 3015-3018. Scholer, M., D. Hovestadt, F. M. Ipavich, and G. Gloeckler (1981), Upstream energetic ions and electrons: Bow shock-associated or magnetospheric origin, J. Geophys. Res., 86, 9040-9046.

Sheldon, R. B., H. E. Spence, J. D. Sullivan, T. A. Fritz, and J. S. Chen (1998), The discovery of trapped energetic electrons in the outer cusp, Geophys. Res. Lett., 25, 1825-1828.

Sibeck, D. G., R. E. Lopez, and E. C. Roelof (1991), Solar wind control of the magnetopause shape, location, and motion, J. Geophys. Res., 96, $5489-5495$.

Smith, C. F., J. L'Heureux, N. F. Ness, M. H. Acuña, L. F. Burlaga, and J. Scheifele (1998), The ACE Magnetic Fields Experiment, Space Sci. Rev., 86, 613-632.

Sotirelis, T., and C.-I. Meng (1999), Magnetopause from pressure balance, J. Geophys. Res., 104, 6889-6898.

Spreiter, J. R., A. L. Summers, and A. Y. Alksne (1966), Hydrodynamic flow around the magnetosphere, Planet. Space Sci., 14, 223-253.

Stone, E. C., A. M. Frandsen, R. A. Mewaldt, E. R. Christian, D. Margolies, J. F. Ormes, and F. Snow (1998), The Advanced Composition Explorer, Space Sci. Rev., 86, 1-22.

Stubbs, T. J. (2002), Ion injection and transport in the high-latitude magnetosphere, Ph.D. thesis, Imperial Coll., London.

Stubbs, T. J., P. Cargill, M. Grande, B. Kellett, M. Lockwood, and C. Perry (2000), Observations of the northern polar cusp with the POLAR spacecraft, in Proceedings of Cluster-II Workshop on Multiscale/Multipoint Plasma Measurements, ESA SP-449, Eur. Space Agency, London.

Stubbs, T. J., M. Lockwood, P. Cargill, J. Fennell, M. Grande, B. Kellett, C. Perry, and A. Rees (2001), Dawn-dusk asymmetry in particles of solar wind origin within the magnetosphere, Ann. Geophys., 19, 1-9.

Stubbs, T. J., P. J. Cargill, M. Lockwood, M. Grande, B. Kellett, and C. Perry (2004), A comparison between ion characteristics observed by the POLAR and DMSP spacecraft in the dayside magnetosphere, Ann. Geophys., 22, 1033-1046.

Svalgaard, L. (1973), Polar cap magnetic variations and their relationship with the interplanetary magnetic sector structure, J. Geophys. Res., 78, 2064-2078.

Trattner, K. J., E. Mobius, M. Scholer, B. Klecker, M. Hilchenbach, and H. Luhr (1994), Statistical-analysis of diffuse ion events upstream of the Earth's bow shock, J. Geophys. Res., 99, 13,389-13,400.

Trattner, K. J., S. A. Fuselier, W. K. Peterson, S. W. Chang, R. Friedel, and M. R. Aellig (2001), Origins of energetic ions in the cusp, J. Geophys. Res., 106, 5967-5976.

Tsyganenko, N. A. (1989), A magnetospheric magnetic field model with the warped tail current sheet, Planet. Space Sci., 37, 5-20.

Tsyganenko, N. A. (1996), Effects of the solar wind conditions on the global magnetospheric configuration as deduced from data-based field models, in Proceedings of the ICS-3 Conference on Substorms, ESA $S P-389$, pp. $181-185$, Eur. Space Agency, Paris.

Tsyganenko, N. A., and C. T. Russell (1999), Magnetic signatures of the distant polar cusps: Observations by Polar and quantitative modeling, J. Geophys. Res., 104, 24,939-24,955.

Wilken, B., W. Weiß, D. Hall, M. Grande, F. Søraas, and J. F. Fennell (1992), Magnetospheric ion composition spectrometer onboard the CRRES spacecraft, J. Spacecr. Rockets, 29, 585-591.

P. J. Cargill, Space and Atmospheric Physics, Blackett Laboratory, Imperial College, London SW7 2BW, UK.

M. Grande, B. J. Kellett, M. Lockwood, and C. H. Perry, Space Science Department, Rutherford Appleton Laboratory, Chilton, Didcot, Oxfordshire OX11 0QX, UK.

T. J. Stubbs, Laboratory for Extraterrestrial Physics, NASA Goddard Space Flight Center, Building 21, Room C216, Mail Code 690.4, Greenbelt, MD 20771, USA. (tstubbs@lepvax.gsfc.nasa.gov) 